\title{
CONDITIONING AN ADDITIVE FUNCTIONAL OF A MARKOV CHAIN TO STAY NONNEGATIVE. II. HITTING A HIGH LEVEL
}

\author{
SAUL D. JACKA, ${ }^{* * *}$ \\ ZORANA LAZIC **** AND \\ JON WARREN, ${ }^{* * * * *}$ University of Warwick
}

\begin{abstract}
Let $\left(X_{t}\right)_{t \geq 0}$ be a continuous-time irreducible Markov chain on a finite state space $E$, let $v: E \rightarrow \mathbb{R} \backslash\{0\}$, and let $\left(\varphi_{t}\right)_{t \geq 0}$ be defined by $\varphi_{t}=\int_{0}^{t} v\left(X_{s}\right) \mathrm{d} s$. We consider the case in which the process $\left(\varphi_{t}\right)_{t \geq 0}$ is oscillating and that in which $\left(\varphi_{t}\right)_{t \geq 0}$ has a negative drift. In each of these cases, we condition the process $\left(X_{t}, \varphi_{t}\right)_{t \geq 0}$ on the event that $\left(\varphi_{t}\right)_{t \geq 0}$ hits level $y$ before hitting 0 and prove weak convergence of the conditioned process as $y \rightarrow \infty$. In addition, we show the relationship between the conditioning of the process $\left(\varphi_{t}\right)_{t \geq 0}$ with a negative drift to oscillate and the conditioning of it to stay nonnegative for a long time, and the relationship between the conditioning of $\left(\varphi_{t}\right)_{t \geq 0}$ with a negative drift to drift to $\infty$ and the conditioning of it to hit large levels before hitting 0 .
\end{abstract}

Keywords: Markov chain; conditional law; weak convergence

2000 Mathematics Subject Classification: Primary 60J27

Secondary 60B 10

\section{Introduction}

Let $\left(X_{t}\right)_{t \geq 0}$ be a continuous-time irreducible Markov chain on a finite state space $E$, let $v$ be a map $v: E \rightarrow \mathbb{R} \backslash\{0\}$, let $\left(\varphi_{t}\right)_{t \geq 0}$ be the additive functional defined by $\varphi_{t}=\varphi+\int_{0}^{t} v\left(X_{s}\right) \mathrm{d} s$, and let $H_{y}, y \in \mathbb{R}$, be the first hitting time of level $y$ by the process $\left(\varphi_{t}\right)_{t \geq 0}$. In a companion paper (Jacka et al. (2005)), we discussed the problem of conditioning the process $\left(X_{t}, \varphi_{t}\right)_{t \geq 0}$ on the event that the process $\left(\varphi_{t}\right)_{t \geq 0}$ stays nonnegative, that is, the event $\left\{H_{0}=\infty\right\}$. In the oscillating case and in the case of the negative drift of the process $\left(\varphi_{t}\right)_{t \geq 0}$, when the event $\left\{H_{0}=\infty\right\}$ is of probability 0 , the process $\left(X_{t}, \varphi_{t}\right)_{t \geq 0}$ can instead be conditioned on some approximation of the event $\left\{H_{0}=\infty\right\}$. In Jacka et al. (2005), we considered the approximation by the events $\left\{H_{0}>T\right\}, T>0$, and proved weak convergence, as $T \rightarrow \infty$, of the process $\left(X_{t}, \varphi_{t}\right)_{t \geq 0}$ conditioned on this approximation.

In this paper, we look at another approximation of the event $\left\{H_{0}=\infty\right\}$ : the approximation by the events $\left\{H_{0}>H_{y}\right\}, y \in \mathbb{R}$. We are again interested in weak convergence, as $y \rightarrow \infty$, of the process $\left(X_{t}, \varphi_{t}\right)_{t \geq 0}$ conditioned on these approximations.

Our motivation comes from Bertoin and Doney (1994). In that work, the authors considered a real-valued random walk $\left\{S_{n}, n \geq 0\right\}$ that does not drift to $\infty$ and conditioned it to stay nonnegative. They discussed two interpretations of this conditioning: one was the conditioning

Received 21 April 2004; revision received 31 May 2005.

* Postal address: Department of Statistics, University of Warwick, Coventry CV4 7AL, UK.

** Email address: s.d.jacka@warwick.ac.uk

*** Email address: z.lazic@warwick.ac.uk

**** Email address: j.warren@warwick.ac.uk 
of $S$ to exceed level $n$ before hitting 0 , and the other was the conditioning of $S$ to be nonnegative up to time $n$. As will be seen, results for our process $\left(X_{t}, \varphi_{t}\right)_{t \geq 0}$, when conditioned on the event $\left\{H_{0}=\infty\right\}$, appear to be analogues of the results for a random walk.

Furthermore, similar to the results obtained in Bertoin and Doney (1994) for a real-valued random walk $\left\{S_{n}, n \geq 0\right\}$ that does not drift to $\infty$, we show that, in the negative-drift case,

(i) taking the limit, as $y \rightarrow \infty$, of conditioning the process $\left(X_{t}, \varphi_{t}\right)_{t \geq 0}$ on $\left\{H_{y}<\infty\right\}$ and then further conditioning on the event $\left\{H_{0}=\infty\right\}$ yields the same result as taking the limit, as $y \rightarrow \infty$, of conditioning $\left(X_{t}, \varphi_{t}\right)_{t \geq 0}$ on the event $\left\{H_{0}>H_{y}\right\}$; and

(ii) conditioning the process $\left(X_{t}, \varphi_{t}\right)_{t \geq 0}$ on the event that the process $\left(\varphi_{t}\right)_{t \geq 0}$ oscillates and then further conditioning on $\left\{H_{0}=\infty\right\}$ yields the same result as taking the limit, as $T \rightarrow \infty$, of conditioning the process $\left(X_{t}, \varphi_{t}\right)_{t \geq 0}$ on $\left\{H_{0}>T\right\}$.

The organization of the paper is as follows. In Section 2, we state the main theorems in the oscillating and negative-drift cases. In Section 3, we calculate the Green's function and the two-sided exit probabilities of the process $\left(X_{t}, \varphi_{t}\right)_{t \geq 0}$ that are needed for the proofs in subsequent sections. In Section 4, we prove the main theorem in the oscillating case. In Section 5, we prove the main theorem in the negative-drift case. Finally, Sections 6 and 7 deal with the negative-drift case of the process $\left(\varphi_{t}\right)_{t \geq 0}$ and give commuting diagrams illustrating the conditioning of the process $\left(X_{t}, \varphi_{t}\right)_{t \geq 0}$ on $\left\{H_{y}<H_{0}\right\}$ and, respectively, $\left\{H_{0}>T\right\}$ listed in (i) and (ii) above.

All the notation in this paper is taken from Jacka et al. (2005).

\section{Main theorems}

We first recall some notation from Jacka et al. (2005).

Let the process $\left(X_{t}, \varphi_{t}\right)$ be as defined in the introduction. Suppose that both $E^{+}=$ $v^{-1}(0, \infty)$ and $E^{-}=v^{-1}(-\infty, 0)$ are nonempty. For any $y \in \mathbb{R}$, let $E_{y}^{+}$and $E_{y}^{-}$be the halfspaces defined by $E_{y}^{+}=(E \times(y, \infty)) \cup\left(E^{+} \times\{y\}\right)$ and $E_{y}^{-}=(E \times(-\infty, y)) \cup\left(E^{-} \times\{y\}\right)$. Let $H_{y}, y \in \mathbb{R}$, be the first crossing time of the level $y$ by the process $\left(\varphi_{t}\right)_{t \geq 0}$, defined by

$$
H_{y}= \begin{cases}\inf \left\{t>0: \varphi_{t}<y\right\} & \text { if }\left(X_{t}, \varphi_{t}\right)_{t \geq 0} \text { starts in } E_{y}^{+}, \\ \inf \left\{t>0: \varphi_{t}>y\right\} & \text { if }\left(X_{t}, \varphi_{t}\right)_{t \geq 0} \text { starts in } E_{y}^{-}\end{cases}
$$

Let $\mathrm{P}_{(e, \varphi)}$ denote the law of the process $\left(X_{t}, \varphi_{t}\right)_{t \geq 0}$ starting at $(e, \varphi)$, and let $\mathrm{E}_{(e, \varphi)}$ denote the expectation operator associated with $\mathrm{P}_{(e, \varphi)}$. Let $\boldsymbol{Q}$ denote the conservative irreducible $Q$-matrix of the process $\left(X_{t}\right)_{t \geq 0}$ and let $\boldsymbol{V}$ be the diagonal matrix $\operatorname{diag}(v(e))$. Let $\boldsymbol{V}^{-1} \boldsymbol{Q} \boldsymbol{\Gamma}=$ $\boldsymbol{\Gamma} \boldsymbol{G}$ be the unique Wiener-Hopf factorization of the matrix $\boldsymbol{V}^{-1} \boldsymbol{Q}$ (see Lemma 3.4 of Jacka $e t$ al. (2005)). Let $\boldsymbol{J}, \boldsymbol{J}_{1}$, and $\boldsymbol{J}_{2}$ be the matrices

$$
\boldsymbol{J}=\left(\begin{array}{cc}
\boldsymbol{I} & \mathbf{0} \\
\mathbf{0} & -\boldsymbol{I}
\end{array}\right), \quad \boldsymbol{J}_{1}=\left(\begin{array}{cc}
\boldsymbol{I} & \mathbf{0} \\
\mathbf{0} & \mathbf{0}
\end{array}\right), \quad \boldsymbol{J}_{2}=\left(\begin{array}{cc}
\mathbf{0} & \mathbf{0} \\
\mathbf{0} & \boldsymbol{I}
\end{array}\right),
$$

and let $\boldsymbol{\Gamma}_{2}$ be a matrix given by $\boldsymbol{\Gamma}_{2}=\boldsymbol{J} \boldsymbol{\Gamma} \boldsymbol{J}$. Throughout, $\boldsymbol{I}$ denotes the identity matrix, and $M(i, j)=[\boldsymbol{M}](i, j)$ is used to denote the $(i, j)$ th entry of a matrix $\boldsymbol{M}$. In $\boldsymbol{J}_{1}$ and the first row of $\boldsymbol{J}, \boldsymbol{I}$ is of order $\left|E^{+}\right|$, while in $\boldsymbol{J}_{2}$ and the second row of $\boldsymbol{J}$, it is of order $\left|E^{-}\right|$. For a fixed $y>0$, let $\mathrm{P}_{(e, \varphi)}^{[y]}$ denote the law of the process $\left(X_{t}, \varphi_{t}\right)_{t \geq 0}$, starting at $(e, \varphi) \in E_{0}^{+}$, conditioned on the event $\left\{H_{y}<H_{0}\right\}$. We are interested in weak convergence of the restriction to $\mathcal{F}_{t}$ of $\left(\mathrm{P}_{(e, \varphi)}^{[y]}\right)_{y \geq 0}$ as $y \rightarrow \infty$. 
Theorem 2.1. Suppose that the process $\left(\varphi_{t}\right)_{t \geq 0}$ oscillates. Then, for a fixed $(e, \varphi) \in E_{0}^{+}$and $t \geq 0$, the restrictions to $\mathcal{F}_{t}$ of the measures $\left(\mathrm{P}_{(e, \varphi)}^{[y]}\right)_{y \geq 0}$ converge weakly, as $y \rightarrow \infty$, to the restriction to $\mathcal{F}_{t}$ of the probability measure $\mathrm{P}_{(e, \varphi)}^{h_{r}}$, which is given by

$$
\mathrm{P}_{(e, \varphi)}^{h_{\boldsymbol{r}}}(A)=\frac{\mathrm{E}_{(e, \varphi)}\left(1(A) h_{\boldsymbol{r}}\left(X_{s}, \varphi_{s}\right) 1\left(s<H_{0}\right)\right)}{h_{\boldsymbol{r}}(e, \varphi)}, \quad s \geq 0, A \in \mathcal{F}_{s},
$$

where $h_{\boldsymbol{r}}$ is a positive, harmonic function for the process $\left(X_{t}, \varphi_{t}\right)_{t \geq 0}$, given by

$$
h_{\boldsymbol{r}}(e, y)=\left[\mathrm{e}^{-y \boldsymbol{V}^{-1} \boldsymbol{Q}} \boldsymbol{J}_{1} \boldsymbol{\Gamma}_{2} \boldsymbol{r}\right](e), \quad(e, y) \in E \times \mathbb{R},
$$

and $\boldsymbol{V}^{-1} \boldsymbol{Q} \boldsymbol{r}=\mathbf{1}$.

By comparing this theorem with Theorem 2.1 of Jacka et al. (2005), we see that the measures $\left(\mathrm{P}_{(e, \varphi)}^{[y]}\right)_{y \geq 0}$ and $\left(\mathrm{P}_{(e, \varphi)}^{(T)}\right)_{T \geq 0}$ converge weakly to the same limit. Therefore, in the oscillating case, conditioning $\left(X_{t}, \varphi_{t}\right)_{t \geq 0}$ on $\left\{H_{y}<H_{0}\right\}, y>0$, and conditioning $\left(X_{t}, \varphi_{t}\right)_{t \geq 0}$ on $\left\{H_{0}>T\right\}, T>0$, yield the same result.

Let $\boldsymbol{f}_{\text {max }}$ be the eigenvector of the matrix $\boldsymbol{V}^{-1} \boldsymbol{Q}$ associated with its eigenvalue with maximal nonpositive real part. In the negative-drift case, the weak limit, as $y \rightarrow \infty$, of the sequence of restrictions to $\mathcal{F}_{t}$ of $\left(\mathrm{P}_{(e, \varphi)}^{[y]}\right)_{y \geq 0}$ is given in the following theorem.

Theorem 2.2. Suppose that the process $\left(\varphi_{t}\right)_{t \geq 0}$ drifts to $-\infty$. Then, for a fixed $(e, \varphi) \in E_{0}^{+}$ and $t \geq 0$, the restrictions to $\mathcal{F}_{t}$ of the measures $\left(\mathrm{P}_{(e, \varphi)}^{[y]}\right)_{y \geq 0}$ converge weakly, as $y \rightarrow \infty$, to the restriction to $\mathcal{F}_{t}$ of the probability measure $\mathrm{P}_{(e, \varphi)}^{h_{f_{\max },} \text {, which is given by }}$

$$
\mathrm{P}_{(e, \varphi)}^{h_{f_{\max }}}(A)=\frac{\mathrm{E}_{(e, \varphi)}\left(1(A) h_{f_{\max }}\left(X_{s}, \varphi_{s}\right) 1\left(s<H_{0}\right)\right)}{h_{f_{\max }}(e, \varphi)}, \quad s \geq 0, A \in \mathcal{F}_{s},
$$

where $h_{f_{\max }}$ is a positive, harmonic function for the process $\left(X_{t}, \varphi_{t}\right)_{t \geq 0}$, given by

$$
h_{f_{\max }}(e, y)=\left[\mathrm{e}^{-y \boldsymbol{V}^{-1} \boldsymbol{Q}} \boldsymbol{J}_{1} \boldsymbol{\Gamma}_{2} \boldsymbol{f}_{\max }\right](e), \quad(e, y) \in E \times \mathbb{R} .
$$

Before we prove Theorems 2.1 and 2.2, we recall some more notation from Jacka et al. (2005) that will be used in the sequel.

The matrices $\boldsymbol{G}^{+}$and $\boldsymbol{G}^{-}$are components of the matrix $\boldsymbol{G}$, i.e.

$$
\boldsymbol{G}=\left(\begin{array}{cc}
\boldsymbol{G}^{+} & \mathbf{0} \\
\mathbf{0} & -\boldsymbol{G}^{-}
\end{array}\right),
$$

and the matrices $\Pi^{+}$and $\Pi^{-}$are components of the matrix $\Gamma$ determined by the Wiener-Hopf factorization of the matrix $\boldsymbol{V}^{-1} \boldsymbol{Q}$, i.e.

$$
\Gamma=\left(\begin{array}{cc}
\boldsymbol{I} & \boldsymbol{\Pi}^{-} \\
\boldsymbol{\Pi}^{+} & \boldsymbol{I}
\end{array}\right)
$$

In other words, the matrix $\boldsymbol{G}^{+}$is the $Q$-matrix of the process $\left(X_{H_{y}}\right)_{y \geq 0},\left(X_{0}, \varphi_{0}\right) \in E^{+} \times\{0\}$, the matrix $G^{-}$is the $Q$-matrix of the process $\left(X_{H_{-y}}\right)_{y \geq 0},\left(X_{0}, \varphi_{0}\right) \in E^{-} \times\{0\}$, and the matrices $\Pi^{-}$and $\Pi^{+}$determine the probability distribution of the process $\left(X_{t}\right)_{t \geq 0}$ at the time $\left(\varphi_{t}\right)_{t \geq 0}$ hits 0 , that is, the probability distribution of $X_{H_{0}}$ (see Lemma 3.4 of Jacka et al. (2005)).

The matrix $\boldsymbol{F}(y), y \in \mathbb{R}$, is defined by

$$
\boldsymbol{F}(y)= \begin{cases}\boldsymbol{J}_{1} \mathrm{e}^{y \boldsymbol{G}}=\mathrm{e}^{y \boldsymbol{G}} \boldsymbol{J}_{1}, & y>0, \\ \boldsymbol{J}_{2} \mathrm{e}^{y \boldsymbol{G}}=\mathrm{e}^{y \boldsymbol{G}} \boldsymbol{J}_{2}, & y<0 .\end{cases}
$$


For any vector $\boldsymbol{g}$ on $E$, let $\boldsymbol{g}^{+}$and $\boldsymbol{g}^{-}$denote its restrictions to $E^{+}$and $E^{-}$, respectively. We write the column vector $\boldsymbol{g}$ as

$$
g=\left(\begin{array}{l}
g^{+} \\
g^{-}
\end{array}\right)
$$

and the row vector $\boldsymbol{\mu}$ as $\boldsymbol{\mu}=\left(\boldsymbol{\mu}^{+}, \boldsymbol{\mu}^{-}\right)$.

A vector $\boldsymbol{g}$ is associated with an eigenvalue $\lambda$ of the matrix $\boldsymbol{V}^{-1} \boldsymbol{Q}$ if there exists a $k \in \mathbb{N}$ such that $\left(\boldsymbol{V}^{-1} \boldsymbol{Q}-\lambda \boldsymbol{I}\right)^{k} \boldsymbol{g}=\mathbf{0}$.

By $\mathcal{B}$ we denote a basis, in the space of all vectors on $E$, containing exactly $n=\left|E^{+}\right|$ vectors, $\boldsymbol{f}_{1}, \boldsymbol{f}_{2}, \ldots, \boldsymbol{f}_{n}$, such that each $\boldsymbol{f}_{j}, j=1, \ldots, n$, is associated with an eigenvalue $\alpha_{j}$ of $\boldsymbol{V}^{-1} \boldsymbol{Q}$ for which $\operatorname{Re}\left(\alpha_{j}\right) \leq 0$, and containing exactly $m=\left|E^{-}\right|$vectors, $\boldsymbol{g}_{1}, \boldsymbol{g}_{2}, \ldots, \boldsymbol{g}_{m}$, such that each $\boldsymbol{g}_{k}, k=1, \ldots, m$, is associated with an eigenvalue $\beta_{k}$ of $\boldsymbol{V}^{-1} \boldsymbol{Q}$ for which $\operatorname{Re}\left(\beta_{k}\right) \geq 0$. The vectors $\boldsymbol{f}_{1}^{+}, \boldsymbol{f}_{2}^{+}, \ldots, \boldsymbol{f}_{n}^{+}$form a basis $\mathcal{N}^{+}$in the space of all vectors on $E^{+}$ and the vectors $\boldsymbol{g}_{1}^{-}, \boldsymbol{g}_{2}^{-}, \ldots, \boldsymbol{g}_{m}^{-}$form a basis $\mathcal{P}^{-}$in the space of all vectors on $E^{-}$.

The matrix $\boldsymbol{V}^{-1} \boldsymbol{Q}$ cannot have strictly imaginary eigenvalues. All eigenvalues of $\boldsymbol{V}^{-1} \boldsymbol{Q}$ with negative and positive real parts respectively coincide with the eigenvalues of $\boldsymbol{G}^{+}$and $-\boldsymbol{G}^{-}$. The matrices $\boldsymbol{G}^{+}$and $\boldsymbol{G}^{-}$are irreducible $Q$-matrices with simple eigenvalues

$$
\begin{aligned}
\alpha_{\max } & :=\max _{1 \leq j \leq n} \operatorname{Re}\left(\alpha_{j}\right) \leq 0, \\
-\beta_{\min } & :=\max _{1 \leq k \leq m} \operatorname{Re}\left(-\beta_{k}\right)=-\min _{1 \leq k \leq m} \operatorname{Re}\left(\beta_{k}\right) \leq 0,
\end{aligned}
$$

respectively, while $\boldsymbol{f}_{\max }$ and $\boldsymbol{g}_{\min }$ are the eigenvectors of the matrix $\boldsymbol{V}^{-1} \boldsymbol{Q}$ respectively associated with its eigenvalues $\alpha_{\max }$ and $\beta_{\min }$. Therefore, $\boldsymbol{f}_{\max }^{+}$and $\boldsymbol{g}_{\min }^{-}$are the PerronFrobenius eigenvectors of the matrices $\boldsymbol{G}^{+}$and $\boldsymbol{G}^{-}$, respectively.

If the process $\left(\varphi_{t}\right)_{t \geq 0}$ drifts to $-\infty$ then $\alpha_{\max }<0$ and $\beta_{\min }=0$; if it drifts to $\infty$ then $\alpha_{\max }=0$ and $\beta_{\min }>0$. If $\left(\varphi_{t}\right)_{t \geq 0}$ oscillates then $\alpha_{\max }=\beta_{\min }=0$ and there exists a vector $\boldsymbol{r}$ such that $\boldsymbol{V}^{-1} \boldsymbol{Q r}=\mathbf{1}$.

\section{The Green's function and hitting probabilities of the process $\left(X_{t}, \varphi_{t}\right)_{t \geq 0}$}

The Green's function of the process $\left(X_{t}, \varphi_{t}\right)_{t \geq 0}$, denoted by $G((e, \varphi),(f, y))$ for any $(e, \varphi),(f, y) \in E \times \mathbb{R}$, is defined as

$$
G((e, \varphi),(f, y))=\mathrm{E}_{(e, \varphi)}\left(\sum_{0 \leq s<\infty} 1\left(X_{s}=f, \varphi_{s}=y\right)\right),
$$

noting that the process $\left(X_{t}, \varphi_{t}\right)_{t \geq 0}$ hits any fixed state at a discrete time. For simplicity of notation, let $\boldsymbol{G}(\varphi, y)$ denote the matrix $(G((\cdot, \varphi),(\cdot, y)))_{E \times E}$.

Theorem 3.1. In the drift cases,

$$
\boldsymbol{G}(0,0)=\boldsymbol{\Gamma}_{2}^{-1}=\left(\begin{array}{cc}
\left(\boldsymbol{I}-\boldsymbol{\Pi}^{-} \boldsymbol{\Pi}^{+}\right)^{-1} & \boldsymbol{\Pi}^{-}\left(\boldsymbol{I}-\boldsymbol{\Pi}^{+} \boldsymbol{\Pi}^{-}\right)^{-1} \\
\boldsymbol{\Pi}^{+}\left(\boldsymbol{I}-\boldsymbol{\Pi}^{-} \boldsymbol{\Pi}^{+}\right)^{-1} & \left(\boldsymbol{I}-\boldsymbol{\Pi}^{+} \boldsymbol{\Pi}^{-}\right)^{-1}
\end{array}\right) .
$$

In the oscillating case, $\boldsymbol{G}(0,0)$ is undefined.

Proof. By the definitions of $\boldsymbol{G}(0,0)$ and the matrices $\boldsymbol{\Pi}^{+}, \boldsymbol{\Pi}^{-}$, and $\boldsymbol{\Gamma}_{2}$, we have

$$
\boldsymbol{G}(0,0)=\sum_{n=0}^{\infty}\left(\begin{array}{cc}
\mathbf{0} & \boldsymbol{\Pi}^{-} \\
\boldsymbol{\Pi}^{+} & \mathbf{0}
\end{array}\right)^{n}=\sum_{n=0}^{\infty}\left(\boldsymbol{I}-\boldsymbol{\Gamma}_{2}\right)^{n} .
$$


Suppose that the process $\left(\varphi_{t}\right)_{t \geq 0}$ drifts to either $\infty$ or $-\infty$. Then, by Equation (3.6) and Lemma 3.5(iv) of Jacka et al. (2005), exactly one of the matrices $\Pi^{+}$and $\Pi^{-}$is strictly substochastic. In addition, the matrix $\Pi^{-} \boldsymbol{\Pi}^{+}$is positive and, thus, primitive. Therefore, the Perron-Frobenius eigenvalue $\lambda$ of $\Pi^{-} \Pi^{+}$satisfies $0<\lambda<1$, which, by the Perron-Frobenius theorem for primitive matrices (see Seneta (1981)), implies that

$$
\lim _{n \rightarrow \infty} \frac{\left(\boldsymbol{\Pi}^{-} \boldsymbol{\Pi}^{+}\right)^{n}}{\lambda^{n}}=\text { const. } \neq \mathbf{0} .
$$

Therefore, $\left(\boldsymbol{\Pi}^{-} \boldsymbol{\Pi}^{+}\right)^{n} \rightarrow 0$ entrywise as $n \rightarrow \infty$ and, similarly, $\left(\boldsymbol{\Pi}^{+} \boldsymbol{\Pi}^{-}\right)^{n} \rightarrow 0$ entrywise as $n \rightarrow \infty$. Hence, $\left(\boldsymbol{I}-\boldsymbol{\Gamma}_{2}\right)^{n} \rightarrow 0$ as $n \rightarrow \infty$. Since

$$
\boldsymbol{I}-\left(\boldsymbol{I}-\boldsymbol{\Gamma}_{2}\right)^{n+1}=\boldsymbol{\Gamma}_{2} \sum_{k=0}^{n}\left(\boldsymbol{I}-\boldsymbol{\Gamma}_{2}\right)^{k}
$$

and, by Lemma 3.5(ii) of Jacka et al. (2005), $\boldsymbol{\Gamma}_{2}^{-1}$ exists, by letting $n \rightarrow \infty$ we obtain

$$
\boldsymbol{G}(0,0)=\sum_{n=0}^{\infty}\left(\boldsymbol{I}-\boldsymbol{\Gamma}_{2}\right)^{n}=\boldsymbol{\Gamma}_{2}^{-1}
$$

Suppose now that the process $\left(\varphi_{t}\right)_{t \geq 0}$ oscillates. Then, again by Equation (3.6) and Lemma 3.5(iv) of Jacka et al. (2005), the matrices $\Pi^{+}$and $\Pi^{-}$are stochastic. Thus, $\left(\boldsymbol{I}-\boldsymbol{\Gamma}_{2}\right) \mathbf{1}=\mathbf{1}$ and

$$
\boldsymbol{G}(0,0) \mathbf{1}=\sum_{n=0}^{\infty}\left(\boldsymbol{I}-\boldsymbol{\Gamma}_{2}\right)^{n} \mathbf{1}=\sum_{n=0}^{\infty} \mathbf{1}
$$

Since the matrix $Q$ is irreducible, it follows that $\boldsymbol{G}(0,0)$ is undefined.

Theorem 3.2. In the drift cases, the Green's function $G((e, \varphi),(f, y))$ of the process $\left(X_{t}, \varphi_{t}\right)_{t \geq 0}$ is given by the $|E| \times|E|$ matrix $\boldsymbol{G}(\varphi, y)$, where

$$
\boldsymbol{G}(\varphi, y)= \begin{cases}\boldsymbol{\Gamma} \boldsymbol{F}(y-\varphi) \boldsymbol{\Gamma}_{2}^{-1}, & \varphi \neq y, \\ \boldsymbol{\Gamma}_{2}^{-1}, & \varphi=y .\end{cases}
$$

Proof. By Theorem 3.1, $\boldsymbol{G}(y, y)=\boldsymbol{G}(0,0)=\boldsymbol{\Gamma}_{2}^{-1}$, and, by Lemma 3.7 of Jacka et al. (2005),

$$
\mathrm{P}_{(e, \varphi-y)}\left(X_{H_{0}}=e^{\prime}, H_{0}<\infty\right)=[\boldsymbol{\Gamma} \boldsymbol{F}(y-\varphi)]\left(e, e^{\prime}\right), \quad \varphi \neq y .
$$

The theorem then follows from

$$
G((e, \varphi),(f, y))=\sum_{e^{\prime} \in E} \mathrm{P}_{(e, \varphi-y)}\left(X_{H_{0}}=e^{\prime}, H_{0}<\infty\right) G\left(\left(e^{\prime}, 0\right),(f, 0)\right) .
$$

The Green's function $G_{0}((e, \varphi),(f, y)),(e, \varphi),(f, y) \in E \times \mathbb{R}$, of the process $\left(X_{t}, \varphi_{t}\right)_{t \geq 0}$ killed when the process $\left(\varphi_{t}\right)_{t \geq 0}$ crosses 0 is defined by

$$
G_{0}((e, \varphi),(f, y))=\mathrm{E}_{(e, \varphi)}\left(\sum_{0 \leq s<H_{0}} 1\left(X_{s}=f, \varphi_{s}=y\right)\right)
$$

(and written $\boldsymbol{G}_{0}(\varphi, y)$ in matrix notation). It follows that $\boldsymbol{G}_{0}(\varphi, y)=\mathbf{0}$ if $\varphi y<0$, that $\boldsymbol{G}_{0}(\varphi, 0)=\mathbf{0}$ if $\varphi \neq 0$, and that $\boldsymbol{G}_{0}(0,0)=\boldsymbol{I}$. To calculate $\boldsymbol{G}_{0}(\varphi, y)$ for $|\varphi| \leq|y|, \varphi y \geq 0$, $y \neq 0$, we use the following lemma. 
Lemma 3.1. Let $(f, y) \in E^{+} \times(0, \infty)$ be fixed and let the process $\left(X_{t}, \varphi_{t}\right)_{t \geq 0}$ start at $(e, \varphi) \in$ $E \times(0, y)$. Let $(e, \varphi) \mapsto h((e, \varphi),(f, y))$ be a bounded function on $E \times(0, y)$ such that the process $\left(h\left(\left(X_{t \wedge H_{0} \wedge H_{y}}, \varphi_{t \wedge H_{0} \wedge H_{y}}\right),(f, y)\right)\right)_{t \geq 0}$ is a uniformly integrable martingale and

$$
\begin{aligned}
& h((e, 0),(f, y))=0, \quad e \in E^{-}, \\
& h((e, y),(f, y))=G_{0}((e, y),(f, y)) .
\end{aligned}
$$

Then

$$
h((e, \varphi),(f, y))=G_{0}((e, \varphi),(f, y)), \quad(e, \varphi) \in E \times(0, y) .
$$

Proof. The proof of the lemma is based on the fact that a uniformly integrable martingale that almost surely hits 0 is almost surely 0 . We leave the reader to supply the details.

Let $\boldsymbol{A}_{y}, \boldsymbol{B}_{y}, \boldsymbol{C}_{y}$, and $\boldsymbol{D}_{y}$ be the components of the matrix $\mathrm{e}^{-y \boldsymbol{V}^{-1} \boldsymbol{Q}}$, such that, for any $y \in \mathbb{R}$,

$$
\mathrm{e}^{-y \boldsymbol{V}^{-1} \boldsymbol{Q}}=\left(\begin{array}{ll}
\boldsymbol{A}_{y} & \boldsymbol{B}_{y} \\
\boldsymbol{C}_{y} & \boldsymbol{D}_{y}
\end{array}\right)
$$

Theorem 3.3. The Green's function $G_{0}((e, \varphi),(f, y)),|\varphi| \leq|y|, \varphi y \geq 0, y \neq 0, e, f \in E$, is given by the following $|E| \times|E|$ matrix $\boldsymbol{G}_{0}(\varphi, y)$ :

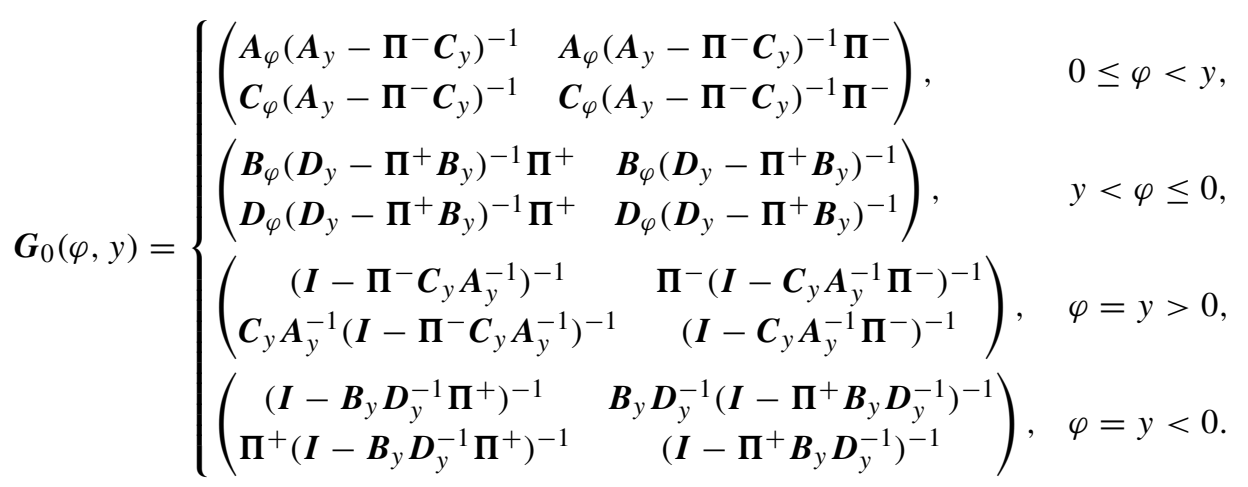

In the drift cases, $\boldsymbol{G}_{0}(\varphi, y)$ is written in matrix notation as

$$
\boldsymbol{G}_{0}(\varphi, y)= \begin{cases}\boldsymbol{\Gamma} \mathrm{e}^{-\varphi} \boldsymbol{G} \boldsymbol{\Gamma}_{2} \boldsymbol{F}(y) \boldsymbol{\Gamma}_{2}^{-1}, & 0 \leq \varphi<y \text { or } y<\varphi \leq 0, \\ \boldsymbol{\Gamma} \boldsymbol{F}(-\varphi) \boldsymbol{\Gamma}_{2} \mathrm{e}^{y \boldsymbol{G}} \boldsymbol{\Gamma}_{2}^{-1}, & 0<y<\varphi \text { or } \varphi<y<0, \\ (\boldsymbol{I}-\boldsymbol{\Gamma} \boldsymbol{F}(-y) \boldsymbol{\Gamma} \boldsymbol{F}(y)) \boldsymbol{\Gamma}_{2}^{-1}, & \varphi=y \neq 0 .\end{cases}
$$

In addition, the Green's function $\boldsymbol{G}_{0}(\varphi, y)$ is positive for all $\varphi, y \in \mathbb{R}$ such that $|y|>0$ and $y \varphi \geq 0$.

Proof. We prove the theorem for $y>0$. The case $y<0$ can be proved in the same way.

Let $y>0$. First we calculate the Green's function $\boldsymbol{G}_{0}(y, y)$. Let $\boldsymbol{Y}_{y}$ denote a matrix on $E^{-} \times E^{+}$with entries

$$
Y_{y}\left(e, e^{\prime}\right)=\mathrm{P}_{(e, y)}\left(X_{H_{y}}=e^{\prime}, H_{y}<H_{0}\right) .
$$

Then

$$
\boldsymbol{G}_{0}(y, y)=\left(\begin{array}{cc}
\boldsymbol{I} & \boldsymbol{\Pi}^{-} \\
\boldsymbol{Y}_{y} & \boldsymbol{I}
\end{array}\right)\left(\begin{array}{cc}
\sum_{n=0}^{\infty}\left(\boldsymbol{\Pi}^{-} \boldsymbol{Y}_{y}\right)^{n} & \mathbf{0} \\
\mathbf{0} & \sum_{n=0}^{\infty}\left(\boldsymbol{Y}_{y} \boldsymbol{\Pi}^{-}\right)^{n}
\end{array}\right)
$$


By Lemma 3.5(vi) of Jacka et al. (2005), the matrix $\boldsymbol{Y}_{y}$ is positive and $\mathbf{0}<\boldsymbol{Y}_{y} \mathbf{1}^{+}<\mathbf{1}^{-}$, componentwise. Hence, $\boldsymbol{\Pi}^{-} \boldsymbol{Y}_{y}$ is positive and therefore irreducible and its Perron-Frobenius eigenvalue $\lambda$ satisfies $0<\lambda<1$. Thus,

$$
\lim _{n \rightarrow \infty} \frac{\left(\boldsymbol{\Pi}^{-} \boldsymbol{Y}_{y}\right)^{n}}{\lambda^{n}}=\text { const. } \neq \mathbf{0},
$$

which implies that $\left(\boldsymbol{\Pi}^{-} \boldsymbol{Y}_{y}\right)^{n} \rightarrow \mathbf{0}$ entrywise as $n \rightarrow \infty$. Similarly, $\left(\boldsymbol{Y}_{y} \boldsymbol{\Pi}^{-}\right)^{n} \rightarrow \mathbf{0}$ entrywise as $n \rightarrow \infty$.

Furthermore, the essentially nonnegative matrices $\boldsymbol{\Pi}^{-} \boldsymbol{Y}_{y}-\boldsymbol{I}$ and $\boldsymbol{Y}_{y} \boldsymbol{\Pi}^{-}-\boldsymbol{I}$ are invertible because their Perron-Frobenius eigenvalues are negative and, by the same argument, the matrices $\left(\boldsymbol{I}-\boldsymbol{\Pi}^{-} \boldsymbol{Y}_{y}\right)^{-1}$ and $\left(\boldsymbol{I}-\boldsymbol{Y}_{y} \boldsymbol{\Pi}^{-}\right)^{-1}$ are positive. Since

$$
\begin{aligned}
& \sum_{k=0}^{n}\left(\boldsymbol{\Pi}^{-} \boldsymbol{Y}_{y}\right)^{k}=\left(\boldsymbol{I}-\boldsymbol{\Pi}^{-} \boldsymbol{Y}_{y}\right)^{-1}\left(\boldsymbol{I}-\left(\boldsymbol{\Pi}^{-} \boldsymbol{Y}_{y}\right)^{n+1}\right), \\
& \sum_{k=0}^{n}\left(\boldsymbol{Y}_{y} \boldsymbol{\Pi}^{-}\right)^{k}=\left(\boldsymbol{I}-\boldsymbol{Y}_{y} \boldsymbol{\Pi}^{-}\right)^{-1}\left(\boldsymbol{I}-\left(\boldsymbol{Y}_{y} \boldsymbol{\Pi}^{-}\right)^{n+1}\right),
\end{aligned}
$$

by letting $n \rightarrow \infty$ we finally obtain

$$
\boldsymbol{G}_{0}(y, y)=\left(\begin{array}{cc}
\left(\boldsymbol{I}-\boldsymbol{\Pi}^{-} \boldsymbol{Y}_{y}\right)^{-1} & \boldsymbol{\Pi}^{-}\left(\boldsymbol{I}-\boldsymbol{\Pi}^{-} \boldsymbol{Y}_{y}\right)^{-1} \\
\boldsymbol{Y}_{y}\left(\boldsymbol{I}-\boldsymbol{Y}_{y} \boldsymbol{\Pi}^{-}\right)^{-1} & \left(\boldsymbol{I}-\boldsymbol{Y}_{y} \boldsymbol{\Pi}^{-}\right)^{-1}
\end{array}\right)=\left(\begin{array}{cc}
\boldsymbol{I} & -\boldsymbol{\Pi}^{-} \\
-\boldsymbol{Y}_{y}^{-1} & \boldsymbol{I}
\end{array}\right)^{-1} .
$$

By Lemma 3.5(i) and (vi) of Jacka et al. (2005), the matrices $\boldsymbol{\Pi}^{-}$and $\boldsymbol{Y}_{y}$ are positive. Since the matrices $\left(\boldsymbol{I}-\boldsymbol{\Pi}^{-} \boldsymbol{Y}_{y}\right)^{-1}$ and $\left(\boldsymbol{I}-\boldsymbol{Y}_{y} \boldsymbol{\Pi}^{-}\right)^{-1}$ are also positive, it follows that $\boldsymbol{G}_{0}(y, y), y>0$, is positive.

We now calculate the Green's function $\boldsymbol{G}_{0}(\varphi, y)$ for $0 \leq \varphi<y$. Let $(f, y) \in E^{+} \times(0, \infty)$ be fixed and let the process $\left(X_{t}, \varphi_{t}\right)_{t \geq 0}$ start in $E \times(0, y)$. Let

$$
h((e, \varphi),(f, y))=\left[\mathrm{e}^{-\varphi \boldsymbol{V}^{-1}} \boldsymbol{Q}_{\left.\boldsymbol{g}_{f, y}\right](e),}\right.
$$

for some vector $\boldsymbol{g}_{f, y}$ on $E$. Since, by Equation (3.4) of Jacka et al. (2005), $g h=0$, the process $\left(h\left(\left(X_{t}, \varphi_{t}\right),(f, y)\right)\right)_{t \geq 0}$ is a local martingale and, because the function $h$ is bounded on every finite interval, it is a martingale. In addition, $\left(h\left(\left(X_{t \wedge H_{0} \wedge H_{y}}, \varphi_{t \wedge H_{0} \wedge H_{y}}\right),(f, y)\right)\right)_{t \geq 0}$ is a bounded martingale and therefore uniformly integrable.

We want the function $h$ to satisfy the boundary conditions in Lemma 3.1. Let $\boldsymbol{h}_{y}(\varphi)$ be an $|E| \times\left|E^{+}\right|$matrix with entries

$$
h_{y}(\varphi)(e, f)=h((e, \varphi),(f, y)) .
$$

Then, from (3.4) and the boundary condition (3.1),

$$
\boldsymbol{h}_{y}(\varphi)=\left(\begin{array}{ll}
\boldsymbol{A}_{\varphi} & \boldsymbol{B}_{\varphi} \\
\boldsymbol{C}_{\varphi} & \boldsymbol{D}_{\varphi}
\end{array}\right)\left(\begin{array}{c}
\boldsymbol{M}_{y} \\
\mathbf{0}
\end{array}\right)=\left(\begin{array}{l}
\boldsymbol{A}_{\varphi} \boldsymbol{M}_{y} \\
\boldsymbol{C}_{\varphi} \boldsymbol{M}_{y}
\end{array}\right), \quad 0 \leq \varphi<y
$$

for some $\left|E^{+}\right| \times\left|E^{+}\right|$matrix $\boldsymbol{M}_{y}$. From the boundary condition (3.2), we have

$$
\boldsymbol{A}_{y} \boldsymbol{M}_{y}=\left(\boldsymbol{I}-\boldsymbol{\Pi}^{-} \boldsymbol{Y}_{y}\right)^{-1} \quad \text { and } \quad \boldsymbol{C}_{y} \boldsymbol{M}_{y}=\boldsymbol{Y}_{y}\left(\boldsymbol{I}-\boldsymbol{\Pi}^{-} \boldsymbol{Y}_{y}\right)^{-1},
$$


which imply that $\boldsymbol{M}_{y}=\left(\boldsymbol{A}_{y}-\boldsymbol{\Pi}^{-} \boldsymbol{C}_{y}\right)^{-1}$ and $\boldsymbol{Y}_{y}=\boldsymbol{C}_{y} \boldsymbol{A}_{y}^{-1}$. Hence,

$$
\boldsymbol{h}_{y}(\varphi)=\left(\begin{array}{l}
\boldsymbol{A}_{\varphi}\left(\boldsymbol{A}_{y}-\boldsymbol{\Pi}^{-} \boldsymbol{C}_{y}\right)^{-1} \\
\boldsymbol{C}_{\varphi}\left(\boldsymbol{A}_{y}-\boldsymbol{\Pi}^{-} \boldsymbol{C}_{y}\right)^{-1}
\end{array}\right), \quad 0 \leq \varphi<y,
$$

and the function $h((e, \varphi),(f, y))$ satisfies the boundary conditions (3.1) and (3.2). Therefore, for $0 \leq \varphi<y, \boldsymbol{G}_{0}(\varphi, y)=\boldsymbol{h}_{y}(\varphi)$ on $E \times E^{+}$and, because $\boldsymbol{G}_{0}(\varphi, y)=\boldsymbol{h}_{y}(\varphi) \boldsymbol{\Pi}^{-}$on $E \times E^{-}$, we have

$$
\boldsymbol{G}_{0}(\varphi, y)=\left(\begin{array}{ll}
\boldsymbol{A}_{\varphi}\left(\boldsymbol{A}_{y}-\boldsymbol{\Pi}^{-} \boldsymbol{C}_{y}\right)^{-1} & \boldsymbol{A}_{\varphi}\left(\boldsymbol{A}_{y}-\boldsymbol{\Pi}^{-} \boldsymbol{C}_{y}\right)^{-1} \boldsymbol{\Pi}^{-} \\
\boldsymbol{C}_{\varphi}\left(\boldsymbol{A}_{y}-\boldsymbol{\Pi}^{-} \boldsymbol{C}_{y}\right)^{-1} & \boldsymbol{C}_{\varphi}\left(\boldsymbol{A}_{y}-\boldsymbol{\Pi}^{-} \boldsymbol{C}_{y}\right)^{-1} \boldsymbol{\Pi}^{-}
\end{array}\right), \quad 0 \leq \varphi<y .
$$

Finally, since $\boldsymbol{G}_{0}(y, y), y>0$, is positive, by irreducibility $\boldsymbol{G}_{0}(\varphi, y)$ is also positive for $0 \leq \varphi<y$.

Lemma 3.2. For $a y \neq 0$ and any $(e, f) \in E \times E$, we have

$$
\begin{aligned}
& \mathrm{P}_{(e, \varphi)}\left(X_{H_{y}}=f, H_{y}<H_{0}\right)=\left[\boldsymbol{G}_{0}(\varphi, y)\left(\boldsymbol{G}_{0}(y, y)\right)^{-1}\right](e, f), \quad 0<|\varphi|<|y|, \\
& \mathrm{P}_{(e, y)}\left(X_{H_{y}}=f, H_{y}<H_{0}\right)=\left[\left(\boldsymbol{I}-\left(\boldsymbol{G}_{0}(y, y)\right)^{-1}\right)\right](e, f) .
\end{aligned}
$$

Proof. By Theorem 3.3, the matrix $\boldsymbol{G}_{0}(y, y)$ is invertible. Therefore, the equalities

$$
\begin{aligned}
& G_{0}((e, \varphi),(f, y))=\sum_{e^{\prime} \in E} \mathrm{P}_{(e, \varphi)}\left(X_{H_{y}}=e^{\prime}, H_{y}<H_{0}\right) G_{0}\left(\left(e^{\prime}, y\right),(f, y)\right), \quad \varphi \neq y \neq 0, \\
& G_{0}((e, y),(f, y))=I(e, f)+\sum_{e^{\prime} \in E} \mathrm{P}_{(e, y)}\left(X_{H_{y}}=e^{\prime}, H_{y}<H_{0}\right) G_{0}\left(\left(e^{\prime}, y\right),(f, y)\right), \quad y \neq 0,
\end{aligned}
$$

prove the lemma.

\section{The oscillating case. Proof of Theorem 2.1}

Let $t \geq 0$ be fixed and let $A \in \mathcal{F}_{t}$. We start by considering the limit of $\mathrm{P}_{(e, \varphi)}^{[y]}(A)$ as $y \rightarrow \infty$. For some $(e, \varphi) \in E_{0}^{+}$and $y>\varphi$, by Lemma 3.5(vi) of Jacka et al. (2005), $\mathrm{P}_{(e, \varphi)}\left(H_{y}<H_{0}\right)>0$ for all $y>0$. Hence, by the Markov property, for any $(e, \varphi) \in E_{0}^{+}$and any $A \in \mathcal{F}_{t}$,

$$
\begin{aligned}
\mathrm{P}_{(e, \varphi)}^{[y]}(A)= & \mathrm{P}_{(e, \varphi)}\left(A \mid H_{y}<H_{0}\right) \\
= & \frac{1}{\mathrm{P}_{(e, \varphi)}\left(H_{y}<H_{0}\right)} \mathrm{E}_{(e, \varphi)}\left(1 ( A ) \left[1\left(t<H_{0} \wedge H_{y}\right) \mathrm{P}_{\left(X_{t}, \varphi_{t}\right)}\left(H_{y}<H_{0}\right)\right.\right. \\
& \left.\left.\quad+1\left(H_{y} \leq t<H_{0}\right)+1\left(H_{y}<H_{0} \leq t\right)\right]\right) .
\end{aligned}
$$

Lemma 4.1. Let $\boldsymbol{r}$ be a vector such that $\boldsymbol{V}^{-1} \boldsymbol{Q r}=1$. Then

$$
\begin{gathered}
h_{\boldsymbol{r}}(e, \varphi) \equiv-\left[\mathrm{e}^{-\varphi \boldsymbol{V}^{-1}} \boldsymbol{Q} \boldsymbol{J}_{1} \boldsymbol{\Gamma}_{2} \boldsymbol{r}\right](e)>0, \quad(e, \varphi) \in E_{0}^{+}, \quad \text { and } \\
\lim _{y \rightarrow \infty} \frac{\mathrm{P}_{\left(e^{\prime}, \varphi^{\prime}\right)}\left(H_{y}<H_{0}\right)}{\mathrm{P}_{(e, \varphi)}\left(H_{y}<H_{0}\right)}=\frac{\left[\mathrm{e}^{\left.-\varphi^{\prime} \boldsymbol{V}^{-1} \boldsymbol{Q} \boldsymbol{J}_{1} \boldsymbol{\Gamma}_{2} \boldsymbol{r}\right]\left(e^{\prime}\right)}\right.}{\left[\mathrm{e}^{\left.-\varphi \boldsymbol{V}^{-1} \boldsymbol{Q} \boldsymbol{J}_{1} \boldsymbol{\Gamma}_{2} \boldsymbol{r}\right](e)}, \quad(e, \varphi),\left(e^{\prime}, \varphi^{\prime}\right) \in E_{0}^{+} .\right.} .
\end{gathered}
$$


Proof. (i) For any $y \in \mathbb{R}$, let the matrices $\boldsymbol{A}_{y}$ and $\boldsymbol{C}_{y}$ be components of the matrix $\mathrm{e}^{-y \boldsymbol{V}^{-1} \boldsymbol{Q}}$ given in (3.3), that is,

$$
\mathrm{e}^{-y \boldsymbol{V}^{-1} \boldsymbol{Q}}=\left(\begin{array}{ll}
\boldsymbol{A}_{y} & \boldsymbol{B}_{y} \\
\boldsymbol{C}_{y} & \boldsymbol{D}_{y}
\end{array}\right)
$$

Then, for any $\varphi \in \mathbb{R}$ and $e \in E$,

$$
h_{\boldsymbol{r}}(e, \varphi)=-\left[\mathrm{e}^{-\varphi \boldsymbol{V}^{-1} \boldsymbol{Q}} \boldsymbol{J}_{1} \boldsymbol{\Gamma}_{2} \boldsymbol{r}\right](e)=-\left[\left(\begin{array}{c}
\boldsymbol{A}_{\varphi}\left(\boldsymbol{r}^{+}-\boldsymbol{\Pi}^{-} \boldsymbol{r}^{-}\right) \\
\boldsymbol{C}_{\varphi}\left(\boldsymbol{r}^{+}-\boldsymbol{\Pi}^{-} \boldsymbol{r}^{-}\right)
\end{array}\right)\right](e) .
$$

The outline of the proof is as follows. We first show that the vector $\boldsymbol{A}_{\varphi}\left(\boldsymbol{r}^{+}-\boldsymbol{\Pi}^{-} \boldsymbol{r}^{-}\right)$has a constant sign by showing that it is a Perron-Frobenius eigenvector of some positive matrix. Then, because

$$
\boldsymbol{C}_{\varphi}\left(\boldsymbol{r}^{+}-\boldsymbol{\Pi}^{-} \boldsymbol{r}^{-}\right)=\boldsymbol{C}_{\varphi} \boldsymbol{A}_{\varphi}^{-1} \boldsymbol{A}_{\varphi}\left(\boldsymbol{r}^{+}-\boldsymbol{\Pi}^{-} \boldsymbol{r}^{-}\right)
$$

and, by our Lemma 3.2, our Theorem 3.3, and Lemma 3.5(vi) of Jacka et al. (2005), the matrix $\boldsymbol{C}_{\varphi} \boldsymbol{A}_{\varphi}^{-1}$ is positive, we conclude that the vector $\boldsymbol{C}_{\varphi}\left(\boldsymbol{r}^{+}-\boldsymbol{\Pi}^{-} \boldsymbol{r}^{-}\right)$has the same constant sign and that the function $h_{r}$ has a constant sign. Finally, by Lemma 4.1(ii) of Jacka et al. (2005), we conclude that $h_{\boldsymbol{r}}$ is always positive.

Therefore, all we have to prove is that the vector $\boldsymbol{A}_{\varphi}\left(\boldsymbol{r}^{+}-\boldsymbol{\Pi}^{-} \boldsymbol{r}^{-}\right)$has a constant sign for any $\varphi \in \mathbb{R}$. Let $\boldsymbol{r}$ be a fixed vector such that $\boldsymbol{V}^{-1} \boldsymbol{Q} \boldsymbol{r}=\mathbf{1}$. Then

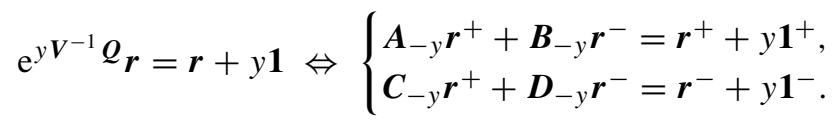

By (3.5), the matrix $\boldsymbol{A}_{\varphi}$ is invertible. Thus, because $\mathbf{1}^{+}=\boldsymbol{\Pi}^{-} \mathbf{1}^{-}$,

$$
\begin{aligned}
& \boldsymbol{A}_{-y}-\boldsymbol{\Pi}^{-} \boldsymbol{C}_{-y}=\left(\boldsymbol{A}_{y}-\boldsymbol{\Pi}^{-} \boldsymbol{C}_{y}\right)^{-1}, \\
& \boldsymbol{B}_{-y}-\boldsymbol{\Pi}^{-} \boldsymbol{D}_{-y}=-\left(\boldsymbol{A}_{-y}-\boldsymbol{\Pi}^{-} \boldsymbol{C}_{-y}\right) \boldsymbol{\Pi}^{-},
\end{aligned}
$$

we have

$$
\left(\boldsymbol{A}_{\varphi}\left(\boldsymbol{A}_{y}-\boldsymbol{\Pi}^{-} \boldsymbol{C}_{y}\right)^{-1} \boldsymbol{A}_{\varphi}^{-1}\right) \boldsymbol{A}_{\varphi}\left(\boldsymbol{r}^{+}-\boldsymbol{\Pi}^{-} \boldsymbol{r}^{-}\right)=\boldsymbol{A}_{\varphi}\left(\boldsymbol{r}^{+}-\boldsymbol{\Pi}^{-} \boldsymbol{r}^{-}\right) .
$$

By Theorem 3.3, the matrix $\boldsymbol{A}_{\varphi}\left(\boldsymbol{A}_{y}-\boldsymbol{\Pi}^{-} \boldsymbol{C}_{y}\right)^{-1}$ is positive for any $\varphi \neq y$. By Lemma 3.2, Theorem 3.3, and by Lemma 3.5(vi) of Jacka et al. (2005), the matrix $\boldsymbol{A}_{\varphi}^{-1}$ is also positive. Hence, the matrix $\boldsymbol{A}_{\varphi}\left(\boldsymbol{A}_{y}-\boldsymbol{\Pi}^{-} \boldsymbol{C}_{y}\right)^{-1} \boldsymbol{A}_{\varphi}^{-1}, \varphi \neq y$, is positive and has a Perron-Frobenius eigenvector of constant sign.

Suppose that $\boldsymbol{A}_{\varphi}\left(\boldsymbol{r}^{+}-\boldsymbol{\Pi}^{-} \boldsymbol{r}^{-}\right)=\mathbf{0}$. Then, because $\boldsymbol{A}_{\varphi}$ is invertible, $\boldsymbol{r}^{+}-\boldsymbol{\Pi}^{-} \boldsymbol{r}^{-}=\mathbf{0}$. If $\boldsymbol{r}^{+}=\boldsymbol{\Pi}^{-} \boldsymbol{r}^{-}$then $\boldsymbol{r}$ is a linear combination of the vectors $\boldsymbol{g}_{k}, k=1, \ldots, m$, in the basis $\mathscr{B}$; however, this is not possible because $\boldsymbol{r}$ is also in the basis $\mathcal{B}$ and is therefore independent of $\boldsymbol{g}_{k}, k=1, \ldots, m$. Hence, $\boldsymbol{A}_{\varphi}\left(\boldsymbol{r}^{+}-\boldsymbol{\Pi}^{-} \boldsymbol{r}^{-}\right) \neq \mathbf{0}$ and, by (4.2), it is the eigenvector of the matrix $\boldsymbol{A}_{\varphi}\left(\boldsymbol{A}_{-y}-\boldsymbol{\Pi}^{-} \boldsymbol{C}_{-y}\right) \boldsymbol{A}_{\varphi}^{-1}$ that corresponds to the eigenvalue 1.

We want to show that the Perron-Frobenius eigenvalue of the matrix

$$
\boldsymbol{A}_{\varphi}\left(\boldsymbol{A}_{-y}-\boldsymbol{\Pi}^{-} \boldsymbol{C}_{-y}\right) \boldsymbol{A}_{\varphi}^{-1}
$$

is 1 . It follows from

$$
\left(\boldsymbol{A}_{\varphi}\left(\boldsymbol{A}_{y}-\boldsymbol{\Pi}^{-} \boldsymbol{C}_{y}\right)^{-1} \boldsymbol{A}_{\varphi}^{-1}\right) \boldsymbol{A}_{\varphi}\left(\boldsymbol{I}-\boldsymbol{\Pi}^{-} \boldsymbol{\Pi}^{+}\right)=\boldsymbol{A}_{\varphi}\left(\boldsymbol{I}-\boldsymbol{\Pi}^{-} \boldsymbol{\Pi}^{+}\right) \mathrm{e}^{y \boldsymbol{G}^{+}}
$$


that if $\alpha$ is a nonzero eigenvalue of the matrix $\boldsymbol{G}^{+}$with some algebraic multiplicity, then $\mathrm{e}^{\alpha y}$ is an eigenvalue of the matrix $\boldsymbol{A}_{\varphi}\left(\boldsymbol{A}_{y}-\boldsymbol{\Pi}^{-} \boldsymbol{C}_{y}\right)^{-1} \boldsymbol{A}_{\varphi}^{-1}$ with the same algebraic multiplicity. Since all $n-1$ nonzero eigenvalues of $\boldsymbol{G}^{+}$have negative real parts, all of the eigenvalues $\mathrm{e}^{\alpha_{j} y}, \alpha_{j} \neq 0, j=1, \ldots, n$, of $\boldsymbol{A}_{\varphi}\left(\boldsymbol{A}_{y}-\boldsymbol{\Pi}^{-} \boldsymbol{C}_{y}\right)^{-1} \boldsymbol{A}_{\varphi}^{-1}$ have real parts strictly less than 1. Thus, 1 is the Perron-Frobenius eigenvalue of the matrix $\boldsymbol{A}_{\varphi}\left(\boldsymbol{A}_{y}-\boldsymbol{\Pi}^{-} \boldsymbol{C}_{y}\right)^{-1} \boldsymbol{A}_{\varphi}^{-1}$, and the vector $\boldsymbol{A}_{\varphi}\left(\boldsymbol{r}^{+}-\boldsymbol{\Pi}^{-} \boldsymbol{r}^{-}\right)$is its Perron-Frobenius eigenvector and therefore has a constant sign.

(ii) The statement of part (ii) follows directly from the equality

$$
\lim _{y \rightarrow \infty} \frac{\mathrm{P}_{\left(e^{\prime}, \varphi^{\prime}\right)}\left(H_{y}<H_{0}\right)}{\mathrm{P}_{(e, \varphi)}\left(H_{y}<H_{0}\right)}=\lim _{y \rightarrow \infty} \frac{\left[\boldsymbol{G}_{0}\left(\varphi^{\prime}, y\right) \mathbf{1}\right]\left(e^{\prime}\right)}{\left[\boldsymbol{G}_{0}(\varphi, y) \mathbf{1}\right](e)},
$$

where $\boldsymbol{G}_{0}(\varphi, y)$ is the Green's function for the killed process defined and determined in Section 3, and from the representation of $\boldsymbol{G}_{0}(\varphi, y)$ given by

$$
\boldsymbol{G}_{0}(\varphi, y) \boldsymbol{1}=\sum_{j, \alpha_{j} \neq 0} a_{j} \mathrm{e}^{-\varphi \boldsymbol{V}^{-1} \boldsymbol{Q}} \boldsymbol{J}_{1} \boldsymbol{\Gamma}_{2} \mathrm{e}^{y \boldsymbol{V}^{-1} \boldsymbol{Q}} \boldsymbol{f}_{j}+c \mathrm{e}^{-\varphi \boldsymbol{V}^{-1} \boldsymbol{Q}} \boldsymbol{J}_{1} \boldsymbol{\Gamma}_{2} \boldsymbol{r},
$$

for some constants $a_{j}, j=1, \ldots, n$, and $c \neq 0$, where the vectors $f_{j}, j=1, \ldots, n$, form part of the basis $\mathcal{B}$ in the space of all vectors on $E$ and are associated with the eigenvalues $\alpha_{j}, j=1, \ldots, n$, of the matrix $\boldsymbol{G}^{+}$. Since $\operatorname{Re}\left(\alpha_{j}\right)<0$ for all $\alpha_{j} \neq 0$, it can be shown that, for every $j$ such that $\alpha_{j} \neq 0, \mathrm{e}^{y V^{-1}} \boldsymbol{Q} \boldsymbol{f}_{j} \rightarrow 0$ as $y \rightarrow \infty$, which proves the statement. For the details of the proof, see Najdanovic (2003).

Proof of Theorem 2.1. By Lemmas 4.1(ii) and 4.3 of Jacka et al. (2005), the function $h_{r}(e, \varphi)$ is positive and harmonic for the process $\left(X_{t}, \varphi_{t}\right)_{t \geq 0}$. Therefore, the measure $\mathrm{P}_{(e, \varphi)}^{h_{r}}$ is well defined.

For fixed $(e, \varphi) \in E_{0}^{+}, t \in[0, \infty)$, and any $y \geq 0$, let $Z_{y}$ be a random variable defined on the probability space $\left(\Omega, \mathcal{F}, \mathrm{P}_{(e, \varphi)}\right)$ by

$$
\begin{aligned}
Z_{y}=\frac{1}{\mathrm{P}_{(e, \varphi)}\left(H_{y}<H_{0}\right)} & \left(1\left(t<H_{0} \wedge H_{y}\right) \mathrm{P}_{\left(X_{t}, \varphi_{t}\right)}\left(H_{y}<H_{0}\right)\right. \\
& \left.+1\left(H_{y} \leq t<H_{0}\right)+1\left(H_{y}<H_{0} \leq t\right)\right) .
\end{aligned}
$$

By our Lemma 4.1(ii) and Lemmas 4.1(ii), 4.2(i), and 4.3 of Jacka et al. (2005), the random variable $Z_{y}$ converges to

$$
\frac{h_{\boldsymbol{r}}\left(X_{t}, \varphi_{t}\right)}{h_{\boldsymbol{r}}(e, \varphi)} 1\left(t<H_{0}\right)
$$

in $L^{1}\left(\Omega, \mathcal{F}, \mathrm{P}_{(e, \varphi)}\right)$ as $y \rightarrow \infty$. Therefore, by (4.1), for a fixed $t \geq 0$ and $A \in \mathcal{F}_{t}$,

$$
\lim _{y \rightarrow \infty} \mathrm{P}_{(e, \varphi)}^{[y]}(A)=\lim _{y \rightarrow \infty} \mathrm{E}_{(e, \varphi)}\left(1(A) Z_{y}\right)=\mathrm{P}_{(e, \varphi)}^{h_{r}}(A),
$$

which, by Lemma 4.2(ii) of Jacka et al. (2005), implies that the restrictions to $\mathcal{F}_{t}$ of the measures $\left(\mathrm{P}_{(e, \varphi)}^{[y]}\right)_{y \geq 0}$ converge weakly to the restriction to $\mathcal{F}_{t}$ of $\mathrm{P}_{(e, \varphi)}^{h_{r}}$ as $y \rightarrow \infty$.

\section{The negative-drift case. Proof of Theorem 2.2}

Again, as in the oscillating case, we start with the limit of $\mathrm{P}_{(e, \varphi)}^{[y]}(A)$, as $y \rightarrow \infty$, by considering

$$
\lim _{y \rightarrow \infty} \frac{\mathrm{P}_{\left(e^{\prime}, \varphi^{\prime}\right)}\left(H_{y}<H_{0}\right)}{\mathrm{P}_{(e, \varphi)}\left(H_{y}<H_{0}\right)} .
$$


First we prove an auxiliary lemma.

Lemma 5.1. For any vector $\boldsymbol{g}$ on $E, \lim _{y \rightarrow \infty} \boldsymbol{F}(y) \boldsymbol{g}=\mathbf{0}$. In addition, for any nonnegative vector $\mathrm{g}$ on $\mathrm{E}$,

$$
\lim _{y \rightarrow \infty} \mathrm{e}^{-\alpha_{\max } y} \boldsymbol{F}(y) \boldsymbol{g}=c \boldsymbol{J}_{1} \boldsymbol{f}_{\max }
$$

for some positive constant $c \in \mathbb{R}$.

Proof. Let

$$
\boldsymbol{g}=\left(\begin{array}{l}
\boldsymbol{g}^{+} \\
\boldsymbol{g}^{-}
\end{array}\right) \quad \text { and } \quad \boldsymbol{g}^{+}=\sum_{j=1}^{n} a_{j} \boldsymbol{f}_{j}^{+},
$$

for some coefficients $a_{j}, j=1, \ldots, n$, where the vectors $f_{j}^{+}, j=1, \ldots, n$, form a basis in the space of all vectors on $E^{+}$and are associated with the eigenvalues $\alpha_{j}, j=1, \ldots, n$, of the matrix $\boldsymbol{G}^{+}$. Then the first equality in the lemma follows from

$$
\boldsymbol{F}(y) \boldsymbol{g}=\left(\begin{array}{cc}
\mathrm{e}^{y \boldsymbol{G}^{+}} & \mathbf{0} \\
\mathbf{0} & \mathbf{0}
\end{array}\right)\left(\begin{array}{l}
\boldsymbol{g}^{+} \\
\boldsymbol{g}^{-}
\end{array}\right)=\left(\begin{array}{c}
\mathrm{e}^{y} \boldsymbol{G}^{+} \boldsymbol{g}^{+} \\
\mathbf{0}
\end{array}\right)=\sum_{j=1}^{n} a_{j}\left(\begin{array}{c}
\mathrm{e}^{y \boldsymbol{G}^{+}} \boldsymbol{f}_{j}^{+} \\
\mathbf{0}
\end{array}\right), \quad y>0
$$

since, for $\operatorname{Re}\left(\alpha_{j}\right)<0, j=1, \ldots, n$, we have $\mathrm{e}^{y \boldsymbol{G}^{+}} \boldsymbol{f}_{j}^{+} \rightarrow 0$ as $y \rightarrow \infty$.

Moreover, by Lemma 3.5(iii) of Jacka et al. (2005), the matrix $\boldsymbol{G}^{+}$is an irreducible $Q$-matrix with Perron-Frobenius eigenvalue $\alpha_{\max }$ and Perron-Frobenius eigenvector $\boldsymbol{f}_{\max }^{+}$. Thus, for any nonnegative vector $\boldsymbol{g}$ on $E^{+}$, by Lemma 3.6(ii) of Jacka et al. (2005),

$$
\lim _{y \rightarrow \infty} \mathrm{e}^{-\alpha_{\max } y}\left[\mathrm{e}^{y \boldsymbol{G}^{+}} \boldsymbol{g}\right](e)=c f_{\max }^{+}(e)
$$

for some positive constant $c \in \mathbb{R}$. Therefore, from (5.1) and (5.2),

$$
\lim _{y \rightarrow \infty} \mathrm{e}^{-\alpha_{\max } y} \boldsymbol{F}(y) \boldsymbol{g}=\lim _{y \rightarrow \infty}\left(\begin{array}{c}
\mathrm{e}^{-\alpha_{\max } y} \mathrm{e}^{y \boldsymbol{G}^{+}} \boldsymbol{g}^{+} \\
\mathbf{0}
\end{array}\right)=c\left(\begin{array}{c}
\boldsymbol{f}_{\max }^{+} \\
\mathbf{0}
\end{array}\right)=c \boldsymbol{J}_{1} \boldsymbol{f}_{\max } .
$$

We now determine the limit

$$
\lim _{y \rightarrow \infty} \frac{\mathrm{P}_{\left(e^{\prime}, \varphi^{\prime}\right)}\left(H_{y}<H_{0}\right)}{\mathrm{P}_{(e, \varphi)}\left(H_{y}<H_{0}\right)} .
$$

Lemma 5.2. For any $(e, \varphi),\left(e^{\prime}, \varphi^{\prime}\right) \in E_{0}^{+}$,

$$
\begin{gathered}
h_{f_{\max }}(e, \varphi):=\left[\mathrm{e}^{-\varphi \boldsymbol{V}^{-1}} \boldsymbol{Q} \boldsymbol{J}_{1} \boldsymbol{\Gamma}_{2} \boldsymbol{f}_{\max }\right](e)>0, \\
\lim _{y \rightarrow \infty} \frac{\mathrm{P}_{\left(e^{\prime}, \varphi^{\prime}\right)}\left(H_{y}<H_{0}\right)}{\mathrm{P}_{(e, \varphi)}\left(H_{y}<H_{0}\right)}=\frac{\left[\mathrm{e}^{-\varphi^{\prime} \boldsymbol{V}^{-1}} \boldsymbol{Q} \boldsymbol{J}_{1} \boldsymbol{\Gamma}_{2} \boldsymbol{f}_{\max }\right]\left(e^{\prime}\right)}{\left[\mathrm{e}^{-\varphi \boldsymbol{V}^{-1} \boldsymbol{Q}} \boldsymbol{J}_{1} \boldsymbol{\Gamma}_{2} \boldsymbol{f}_{\max }\right](e)} .
\end{gathered}
$$

Proof. (i) The function $h_{f_{\max }}$ can be rewritten as

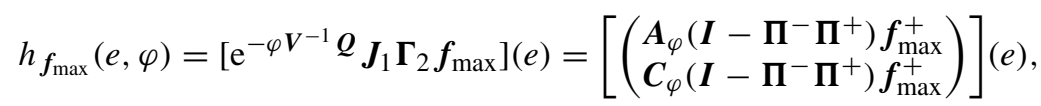

where $\boldsymbol{A}_{\varphi}$ and $\boldsymbol{C}_{\varphi}$ are as given in (3.3). 
We first show that the vector $\boldsymbol{A}_{\varphi}\left(\boldsymbol{I}-\boldsymbol{\Pi}^{-} \boldsymbol{\Pi}^{+}\right) \boldsymbol{f}_{\max }^{+}$is positive. By (3.5), the matrix $\boldsymbol{A}_{\varphi}$ is invertible and, by Equation (3.6) and Lemma 3.5, parts (ii) and (iv), of Jacka et al. (2005), the matrix $\boldsymbol{I}-\boldsymbol{\Pi}^{-} \boldsymbol{\Pi}^{+}$is invertible. Therefore,

$$
\boldsymbol{A}_{\varphi}\left(\boldsymbol{A}_{-y}-\boldsymbol{\Pi}^{-} \boldsymbol{C}_{-y}\right) \boldsymbol{A}_{\varphi}^{-1}=\boldsymbol{A}_{\varphi}\left(\boldsymbol{I}-\boldsymbol{\Pi}^{-} \boldsymbol{\Pi}^{+}\right) \mathrm{e}^{y \boldsymbol{G}^{+}}\left(\boldsymbol{I}-\boldsymbol{\Pi}^{-} \boldsymbol{\Pi}^{+}\right)^{-1} \boldsymbol{A}_{\varphi}^{-1} .
$$

By Theorem 3.3, the matrix $\boldsymbol{A}_{\varphi}\left(\boldsymbol{A}_{y}-\boldsymbol{\Pi}^{-} \boldsymbol{C}_{y}\right)^{-1}, \varphi \neq y$, is positive and, by Lemma 3.2, Theorem 3.3, and by Lemma 3.5(vi) of Jacka et al. (2005), the matrix $\boldsymbol{A}_{\varphi}^{-1}$ is also positive. Hence, the matrix $\boldsymbol{A}_{\varphi}\left(\boldsymbol{A}_{-y}-\boldsymbol{\Pi}^{-} \boldsymbol{C}_{-y}\right) \boldsymbol{A}_{\varphi}^{-1}, \varphi \neq y$, is positive and is similar to $\mathrm{e}^{y \boldsymbol{G}^{+}}$. Thus, $\boldsymbol{A}_{\varphi}\left(\boldsymbol{A}_{-y}-\boldsymbol{\Pi}^{-} \boldsymbol{C}_{-y}\right) \boldsymbol{A}_{\varphi}^{-1}$ and $\mathrm{e}^{y \boldsymbol{G}^{+}}$have the same Perron-Frobenius eigenvalue and, because the Perron-Frobenius eigenvector of $\mathrm{e}^{y G^{+}}$is $\boldsymbol{f}_{\max }^{+}$, it follows that $\boldsymbol{A}_{\varphi}\left(\boldsymbol{I}-\boldsymbol{\Pi}^{-} \boldsymbol{\Pi}^{+}\right) \boldsymbol{f}_{\max }^{+}$is the Perron-Frobenius eigenvector of $\boldsymbol{A}_{\varphi}\left(\boldsymbol{A}_{-y}-\boldsymbol{\Pi}^{-} \boldsymbol{C}_{-y}\right) \boldsymbol{A}_{\varphi}^{-1}$ and, therefore, positive. In addition,

$$
\boldsymbol{C}_{\varphi}\left(\boldsymbol{I}-\boldsymbol{\Pi}^{-} \boldsymbol{\Pi}^{+}\right) \boldsymbol{f}_{\max }^{+}=\boldsymbol{C}_{\varphi} \boldsymbol{A}_{\varphi}^{-1} \boldsymbol{A}_{\varphi}\left(\boldsymbol{I}-\boldsymbol{\Pi}^{-} \boldsymbol{\Pi}^{+}\right) \boldsymbol{f}_{\max }^{+}
$$

and, by Lemma 3.2, Theorem 3.3, and by Lemma 3.5(vi) of Jacka et al. (2005), the matrix $\boldsymbol{C}_{\varphi} \boldsymbol{A}_{\varphi}^{-1}$ is positive. Therefore, the function $h_{f_{\max }}$ is positive.

(ii) By Lemmas 3.2 and 5.1 and Theorem 3.3,

$$
\lim _{y \rightarrow \infty} \frac{\mathrm{P}_{\left(e^{\prime}, \varphi^{\prime}\right)}\left(H_{y}<H_{0}\right)}{\mathrm{P}_{(e, \varphi)}\left(H_{y}<H_{0}\right)}=\lim _{y \rightarrow \infty} \frac{\left[\mathrm{e}^{-\varphi^{\prime} \boldsymbol{V}^{-1} \boldsymbol{Q}} \boldsymbol{\Gamma} \boldsymbol{\Gamma}_{2} \boldsymbol{F}(y) \mathbf{1}\right]\left(e^{\prime}\right)}{\left[\mathrm{e}^{-\varphi \boldsymbol{V}^{-1} \boldsymbol{Q}} \boldsymbol{\Gamma} \boldsymbol{\Gamma}_{2} \boldsymbol{F}(y) \mathbf{1}\right](e)} .
$$

Since the vector $\mathbf{1}$ is nonnegative and $\Gamma \boldsymbol{\Gamma}_{2} \boldsymbol{J}_{1} \boldsymbol{f}_{\max }=\boldsymbol{J}_{1} \boldsymbol{\Gamma}_{2} \boldsymbol{f}_{\max }$, the statement of the lemma follows from Lemma 5.1.

The function $h_{f_{\max }}$ has the property that the process $\left(h_{f_{\max }}\left(X_{t}, \varphi_{t}\right) 1\left(t<H_{0}\right)\right)_{t \geq 0}$ is a martingale under $\mathrm{P}_{(e, \varphi)}$. We prove this in the following lemma.

Lemma 5.3. The function $h_{f_{\max }}(e, \varphi)$ is harmonic for the process $\left(X_{t}, \varphi_{t}\right)_{t \geq 0}$ and the process $\left(h_{f_{\max }}\left(X_{t}, \varphi_{t}\right) 1\left(t<H_{0}\right)\right)_{t \geq 0}$ is a martingale under $\mathrm{P}_{(e, \varphi)}$.

Proof. The function $h_{f_{\max }}(e, \varphi)$ is continuously differentiable in $\varphi$ and, therefore, by Equation (3.4) of Jacka et al. (2005), it is in the domain of the infinitesimal generator $g$ of the process $\left(X_{t}, \varphi_{t}\right)_{t \geq 0}$, with $g h_{f_{\max }}=0$. Thus, the function $h_{f_{\max }}(e, \varphi)$ is harmonic for the process $\left(X_{t}, \varphi_{t}\right)_{t \geq 0}$ and the process $\left(h_{f_{\max }}\left(X_{t}, \varphi_{t}\right)\right)_{t \geq 0}$ is a local martingale under $\mathrm{P}_{(e, \varphi)}$. It follows that the process $\left(h_{f_{\max }}\left(X_{t \wedge H_{0}}, \varphi_{t \wedge H_{0}}\right)=h_{f_{\max }}\left(X_{t}, \varphi_{t}\right) 1\left(t<H_{0}\right)\right)_{t \geq 0}$ is also a local martingale under $\mathrm{P}_{(e, \varphi)}$ and, because it is bounded on every finite interval, is a martingale.

Proof of Theorem 2.2. The proof is exactly the same as the proof of Theorem 2.1 with the function $h_{f_{\max }}$ substituting for $h_{r}$. (We therefore appeal to Lemma 5.2 rather than to Lemma 4.1 for the desired properties of $h_{f_{\max }}$.)

\section{The negative-drift case: conditioning $\left(\varphi_{t}\right)_{t \geq 0}$ to drift to $\infty$}

The process $\left(X_{t}, \varphi_{t}\right)_{t \geq 0}$ can also be conditioned first on the event that $\left(\varphi_{t}\right)_{t \geq 0}$ hits large levels $y$ despite possibly crossing 0 (that is, taking the limit, as $y \rightarrow \infty$, of conditioning $\left(X_{t}, \varphi_{t}\right)_{t \geq 0}$ on $\left\{H_{y}<\infty\right\}$ ), and then the resulting process can be conditioned on the event that $\left(\varphi_{t}\right)_{t \geq 0}$ stays nonnegative. In this section, we show that these two conditionings, performed in the order stated, yield the same result as taking the limit, as $y \rightarrow \infty$, of conditioning $\left(X_{t}, \varphi_{t}\right)_{t \geq 0}$ on $\left\{H_{y}<H_{0}\right\}$. 
Let $(e, \varphi) \in E_{0}^{+}$and $y>\varphi$. Then, by Lemma 3.5(vii) of Jacka et al. (2005), the event $\left\{H_{y}<\infty\right\}$ is of positive probability and the process $\left(X_{t}, \varphi_{t}\right)_{t \geq 0}$ can be conditioned on $\left\{H_{y}<\infty\right\}$ in the standard way. For a fixed $t \geq 0$ and any $A \in \mathcal{F}_{t}$,

$$
\mathrm{P}_{(e, \varphi)}\left(A \mid H_{y}<\infty\right)=\frac{\mathrm{E}_{(e, \varphi)}\left(1(A) \mathrm{P}_{\left(X_{t}, \varphi_{t}\right)}\left(H_{y}<\infty\right) 1\left(t<H_{y}\right)+1(A) 1\left(H_{y}<t\right)\right)}{\mathrm{P}_{(e, \varphi)}\left(H_{y}<\infty\right)}
$$

Lemma 6.1. For any $(e, \varphi),\left(e^{\prime}, \varphi^{\prime}\right) \in E_{0}^{+}$,

$$
\lim _{y \rightarrow \infty} \frac{\mathrm{P}_{\left(e^{\prime}, \varphi^{\prime}\right)}\left(H_{y}<\infty\right)}{\mathrm{P}_{(e, \varphi)}\left(H_{y}<\infty\right)}=\frac{\mathrm{e}^{-\alpha_{\max } \varphi^{\prime}} f_{\max }\left(e^{\prime}\right)}{\mathrm{e}^{-\alpha_{\max } \varphi} f_{\max }(e)} .
$$

Proof. By Lemma 3.7 of Jacka et al. (2005), for $0 \leq \varphi<y$ we have

$$
\mathrm{P}_{(e, \varphi)}\left(H_{y}<\infty\right)=\mathrm{P}_{(e, \varphi-y)}\left(H_{0}<\infty\right)=[\boldsymbol{\Gamma} \boldsymbol{F}(y-\varphi) \mathbf{1}](e) .
$$

The vector $\mathbf{1}$ is nonnegative; hence, by Lemma 5.1 and because $\Gamma \boldsymbol{J}_{1} f_{\max }=f_{\max }$, we have

$$
\begin{aligned}
\lim _{y \rightarrow \infty} \frac{\mathrm{P}_{\left(e^{\prime}, \varphi^{\prime}\right)}\left(H_{y}<\infty\right)}{\mathrm{P}_{(e, \varphi)}\left(H_{y}<\infty\right)} & =\lim _{y \rightarrow \infty} \frac{\mathrm{e}^{-\alpha_{\max } \varphi^{\prime}}\left[\boldsymbol{\Gamma} \mathrm{e}^{-\alpha_{\max }\left(y-\varphi^{\prime}\right)} \boldsymbol{F}(y-\varphi) \mathbf{1}\right]\left(e^{\prime}\right)}{\mathrm{e}^{-\alpha_{\max } \varphi}\left[\boldsymbol{\Gamma} \mathrm{e}^{-\alpha_{\max }(y-\varphi)} \boldsymbol{F}(y-\varphi) \mathbf{1}\right](e)} \\
& =\frac{\mathrm{e}^{-\alpha_{\max } \varphi^{\prime}} f_{\max }\left(e^{\prime}\right)}{\mathrm{e}^{-\alpha_{\max } \varphi} f_{\max }(e)}
\end{aligned}
$$

Let $h_{\max }(e, \varphi)$ be a function on $E \times \mathbb{R}$ defined by

$$
h_{\max }(e, \varphi)=\mathrm{e}^{-\alpha_{\max } \varphi} f_{\max }(e) .
$$

Lemma 6.2. The function $h_{\max }(e, \varphi)$ is harmonic for the process $\left(X_{t}, \varphi_{t}\right)_{t \geq 0}$, and the process $\left(h_{\max }\left(X_{t}, \varphi_{t}\right)\right)_{t \geq 0}$ is a martingale under $\mathrm{P}_{(e, \varphi)}$.

Proof. The function $h_{\max }(e, \varphi)$ is continuously differentiable in $\varphi$ and, therefore, by Equation (3.4) of Jacka et al. (2005), it is in the domain of the infinitesimal generator $g$ of the process $\left(X_{t}, \varphi_{t}\right)_{t \geq 0}$, with $g h_{\max }=0$. It follows that the function $h_{\max }(e, \varphi)$ is harmonic for the process $\left(X_{t}, \varphi_{t}\right)_{t \geq 0}$ and that the process $\left(h_{\max }\left(X_{t}, \varphi_{t}\right)\right)_{t \geq 0}$ is a local martingale under $\mathrm{P}_{(e, \varphi)}$. Since the function $h_{\max }(e, \varphi)$ is bounded on every finite interval, the process $\left(h_{\max }\left(X_{t}, \varphi_{t}\right)\right)_{t \geq 0}$ is a martingale under $\mathrm{P}_{(e, \varphi)}$.

By Lemmas 6.1 and 6.2, we can prove the following theorem.

Theorem 6.1. For a fixed $(e, \varphi) \in E_{0}^{+}$, let $\mathrm{P}_{(e, \varphi)}^{h_{\max }}$ be a measure defined by

$$
\mathrm{P}_{(e, \varphi)}^{h_{\max }}(A)=\frac{\mathrm{E}_{(e, \varphi)}\left(1(A) h_{\max }\left(X_{t}, \varphi_{t}\right)\right)}{h_{\max }(e, \varphi)}, \quad t \geq 0, A \in \mathcal{F}_{t} .
$$

Then $\mathrm{P}_{(e, \varphi)}^{h_{\max }}$ is a probability measure and, for a fixed $t \geq 0$,

$$
\lim _{y \rightarrow \infty} \mathrm{P}_{(e, \varphi)}\left(A \mid H_{y}<\infty\right)=\mathrm{P}_{(e, \varphi)}^{h_{\max }}(A), \quad A \in \mathcal{F}_{t}
$$

Proof. By definition, the function $h_{\max }$ is positive. By Lemma 6.2, it is harmonic for the process $\left(X_{t}, \varphi_{t}\right)_{t \geq 0}$ and the process $\left(h_{\max }\left(X_{t}, \varphi_{t}\right)\right)_{t \geq 0}$ is a martingale under $\mathrm{P}_{(e, \varphi)}$. Hence, $\mathrm{P}_{(e, \varphi)}^{h_{\max }}$ is a probability measure. 
For fixed $(e, \varphi) \in E_{0}^{+}, t \geq 0$, and any $y \geq 0$, let $Z_{y}$ be a random variable defined on the probability space $\left(\Omega, \mathcal{F}, \mathrm{P}_{(e, \varphi)}\right)$ by

$$
Z_{y}=\frac{\mathrm{P}_{\left(X_{t}, \varphi_{t}\right)}\left(H_{y}<\infty\right) 1\left(t<H_{y}\right)+1\left(H_{y}<t\right)}{\mathrm{P}_{(e, \varphi)}\left(H_{y}<\infty\right)} .
$$

By our Lemma 6.1 and Lemmas 4.2(i) and 4.3 of Jacka et al. (2005), the random variable $Z_{y}$ converges to $h_{\max }\left(X_{t}, \varphi_{t}\right) / h_{\max }(e, \varphi)$ in $L^{1}\left(\Omega, \mathcal{F}, \mathrm{P}_{(e, \varphi)}\right)$ as $y \rightarrow \infty$. Therefore, by (6.1), for a fixed $t \geq 0$ and $A \in \mathcal{F}_{t}$, we have

$$
\lim _{y \rightarrow \infty} \mathrm{P}_{(e, \varphi)}\left(A \mid H_{y}<\infty\right)=\lim _{y \rightarrow \infty} \mathrm{E}_{(e, \varphi)}\left(1(A) Z_{y}\right)=\mathrm{P}_{(e, \varphi)}^{h_{\max }}(A) .
$$

We now want to condition the process $\left(X_{t}, \varphi_{t}\right)_{t \geq 0}$, under $\mathrm{P}_{(e, \varphi)}^{h_{\max }}$, on the event $\left\{H_{0}=\infty\right\}$. By Theorem 7.1, $\left(X_{t}\right)_{t \geq 0}$ is a Markov process under $\mathrm{P}_{(e, \varphi)}^{h_{\max }}$ with irreducible conservative $Q$-matrix $Q^{h_{\max }}$ given by

$$
Q^{h_{\max }}\left(e, e^{\prime}\right)=\frac{f_{\max }\left(e^{\prime}\right)}{f_{\max }(e)}\left[\boldsymbol{Q}-\alpha_{\max } \boldsymbol{V}\right]\left(e, e^{\prime}\right), \quad e, e^{\prime} \in E,
$$

and, by the same theorem, the process $\left(\varphi_{t}\right)_{t \geq 0}$ drifts to $\infty$ under $\mathrm{P}_{(e, \varphi)}^{h_{\max }}$. We next find the Wiener-Hopf factorization of the matrix $\boldsymbol{V}^{-1} \boldsymbol{Q}^{h_{\max }}$.

Lemma 6.3. The unique Wiener-Hopf factorization of the matrix $\boldsymbol{V}^{-1} \boldsymbol{Q}^{h_{\max }}$ is given by

$$
\boldsymbol{V}^{-1} \boldsymbol{Q}^{h_{\max }} \boldsymbol{\Gamma}^{h_{\max }}=\boldsymbol{\Gamma}^{h_{\max }} \boldsymbol{G}^{h_{\max }},
$$

where, for any $\left(e, e^{\prime}\right) \in E \times E$,

$$
G^{h_{\max }}\left(e, e^{\prime}\right)=\frac{f_{\max }\left(e^{\prime}\right)}{f_{\max }(e)}\left[\boldsymbol{G}-\alpha_{\max } \boldsymbol{I}\right]\left(e, e^{\prime}\right) \quad \text { and } \quad \Gamma^{h_{\max }}\left(e, e^{\prime}\right)=\frac{f_{\max }\left(e^{\prime}\right)}{f_{\max }(e)} \Gamma\left(e, e^{\prime}\right) .
$$

In addition, if

$$
\boldsymbol{G}^{h_{\max }}=\left(\begin{array}{cc}
\boldsymbol{G}^{h_{\max },+} & \mathbf{0} \\
\mathbf{0} & -\boldsymbol{G}^{h_{\max },-}
\end{array}\right) \quad \text { and } \quad \boldsymbol{\Gamma}^{h_{\max }}=\left(\begin{array}{cc}
\boldsymbol{I} & \boldsymbol{\Pi}^{h_{\max },-} \\
\boldsymbol{\Pi}^{h_{\max },+} & \boldsymbol{I}
\end{array}\right),
$$

then $\boldsymbol{G}^{h_{\max },+}$ is a conservative $Q$-matrix, $\boldsymbol{\Pi}^{h_{\max },+}$ is stochastic, $\boldsymbol{G}^{h_{\max },-}$ is not a conservative $Q$-matrix, and $\Pi^{h_{\max },-}$ is strictly substochastic.

Proof. By definition, the matrices $\boldsymbol{G}^{h_{\max },+}$ and $\boldsymbol{G}^{h_{\max },-}$ are essentially nonnegative. In addition, for any $e \in E^{+},\left[\boldsymbol{G}^{h_{\max },+} \mathbf{1}\right](e)=0$. Hence, $\boldsymbol{G}^{h_{\max },+}$ is a conservative $Q$-matrix. By Lemma 5.2(i),

$$
\boldsymbol{h}_{\boldsymbol{f}_{\max }}^{-}=\left(\boldsymbol{\Pi}^{+} \mathrm{e}^{-\varphi \boldsymbol{G}^{+}}-\mathrm{e}^{\varphi \boldsymbol{G}^{-}} \boldsymbol{\Pi}^{+}\right) \boldsymbol{f}_{\max }^{+}=\mathrm{e}^{-\alpha_{\max } \varphi}\left(\boldsymbol{I}-\mathrm{e}^{\varphi\left(\boldsymbol{G}^{-}+\alpha_{\max } \boldsymbol{I}\right)}\right) \boldsymbol{f}_{\max }^{-}>\mathbf{0} .
$$

Since

$$
\lim _{\varphi \rightarrow 0} \frac{\left(\boldsymbol{I}-\mathrm{e}^{\varphi\left(\boldsymbol{G}^{-}+\alpha_{\max } \boldsymbol{I}\right)}\right) \boldsymbol{f}_{\max }^{-}}{\varphi}=-\left(\boldsymbol{G}^{-}+\alpha_{\max } \boldsymbol{I}\right) \boldsymbol{f}_{\max }^{-}
$$

and $\left(\boldsymbol{I}-\mathrm{e}^{\varphi\left(\boldsymbol{G}^{-}+\alpha_{\max } \boldsymbol{I}\right)}\right) \boldsymbol{f}_{\max }^{-}>\mathbf{0}$, it follows that $\left(\boldsymbol{G}^{-}+\alpha_{\max } \boldsymbol{I}\right) \boldsymbol{f}_{\max }^{-} \leq \mathbf{0}$. Thus, $\boldsymbol{G}^{h_{\max },{ }^{-} \mathbf{1}^{-}} \leq \mathbf{0}$ and, so, $\boldsymbol{G}^{h_{\max },-}$ is a $Q$-matrix. Moreover, if $\left(\boldsymbol{G}^{-}+\alpha_{\max } \boldsymbol{I}\right) \boldsymbol{f}_{\max }^{-}=\mathbf{0}$ then $h_{\boldsymbol{f}_{\max }}(e, \varphi)=0$ 
for $e \in E^{-}$, which is a contradiction of Lemma 5.2. Therefore, the matrix $\boldsymbol{G}^{h_{\max },-}$ is not conservative.

The matrices $\boldsymbol{G}^{h_{\max }}$ and $\boldsymbol{\Gamma}^{h_{\max }}$ satisfy the equality

$$
\boldsymbol{V}^{-1} \boldsymbol{Q}^{h_{\max }} \boldsymbol{\Gamma}^{h_{\max }}=\boldsymbol{\Gamma}^{h_{\max }} \boldsymbol{G}^{h_{\max }},
$$

which, by Lemma 3.4 of Jacka et al. (2005), gives the unique Wiener-Hopf factorization of the matrix $\boldsymbol{V}^{-1} \boldsymbol{Q}^{h_{\max }}$. Furthermore, by Lemma 3.5(iv) of Jacka et al. (2005), $\boldsymbol{\Pi}^{h_{\max },+}$ is a stochastic matrix and $\Pi^{h_{\max },-}$ is a strictly substochastic matrix.

Finally, we prove the main result in this section.

Theorem 6.2. Let $\mathrm{P}_{(e, \varphi)}^{h_{f_{\max }}}$ be as defined in Theorem 2.2. Then, for any $(e, \varphi) \in E_{0}^{+}$and any $t \geq 0$,

$$
\mathrm{P}_{(e, \varphi)}^{h_{\max }}\left(A \mid H_{0}=\infty\right)=\mathrm{P}_{(e, \varphi)}^{h_{f_{\max }}}(A), \quad A \in \mathcal{F}_{t} .
$$

Proof. As we shall see in Section 7, Theorem 7.1 states that the process $\left(\varphi_{t}\right)_{t \geq 0}$, under $\mathrm{P}_{(e, \varphi)}^{h_{\max }}$, drifts to $\infty$. Since, in the positive-drift case, the event $\left\{H_{0}=\infty\right\}$ is of positive probability, for any $t \geq 0$ and any $A \in \mathcal{F}_{t}$ we have

$$
\mathrm{P}_{(e, \varphi)}^{h_{\max }}\left(A \mid H_{0}=\infty\right)=\frac{\mathrm{E}_{(e, \varphi)}^{h_{\max }}\left(1(A) \mathrm{P}_{\left(X_{t}, \varphi_{t}\right)}^{h_{\max }}\left(H_{0}=\infty\right) 1\left(t<H_{0}\right)\right)}{\mathrm{P}_{(e, \varphi)}^{h_{\max }}\left(H_{0}=\infty\right)},
$$

where $\mathrm{E}_{(e, \varphi)}^{h_{\max }}$ denotes the expectation operator associated with the measure $\mathrm{P}_{(e, \varphi)}^{h_{\max }}$.

By Lemma 3.7 of Jacka et al. (2005) and by our Lemma 6.3, for $\varphi>0$ we have

$$
\begin{aligned}
\mathrm{P}_{(e, \varphi)}^{h_{\max }}\left(H_{0}=\infty\right) & =1-\frac{\mathrm{e}^{\alpha_{\max } \varphi}}{f_{\max }(e)} \sum_{e^{\prime} \in E}\left[\boldsymbol{\Gamma} \mathrm{e}^{-\varphi \boldsymbol{G}}\right]\left(e, e^{\prime}\right)\left[\boldsymbol{J}_{2} \mathbf{1}\right]\left(e^{\prime}\right) f_{\max }\left(e^{\prime}\right) \\
& =\frac{1}{h_{\max }(e, \varphi)}\left[\mathrm{e}^{-\alpha_{\max } \varphi} \boldsymbol{f}_{\max }-\boldsymbol{\Gamma} \boldsymbol{F}(-\varphi) \boldsymbol{f}_{\max }\right](e) \\
& =\frac{h_{f_{\max }}(e, \varphi)}{h_{\max }(e, \varphi)},
\end{aligned}
$$

where $h_{f_{\max }}$ is as defined in Lemma 5.2. Similarly, for $e \in E^{+}$,

$$
\mathrm{P}_{(e, 0)}^{h_{\max }}\left(H_{0}=\infty\right)=\frac{\left[\boldsymbol{f}_{\max }^{+}-\boldsymbol{\Pi}^{-} \boldsymbol{f}_{\max }^{-}\right](e)}{f_{\max }^{+}(e)}=\frac{h_{f_{\max }}(e, 0)}{h_{\max }(e, 0)} .
$$

Therefore, the statement of the theorem follows from Theorem 6.1, (6.2), and (6.3).

Let us summarize the results of this section. In the negative-drift case, making the $h$-transform of the process $\left(X_{t}, \varphi_{t}\right)_{t \geq 0}$ by the function $h_{\max }(e, \varphi)=\mathrm{e}^{-\alpha_{\max } \varphi} f_{\max }(e)$ yields the probability measure $\mathrm{P}_{(e, \varphi)}^{h_{\max }}$ under which $\left(X_{t}\right)_{t \geq 0}$ is a Markov process and $\left(\varphi_{t}\right)_{t \geq 0}$ has a positive drift. The process $\left(X_{t}, \varphi_{t}\right)_{t \geq 0}$, under $\mathrm{P}_{(e, \varphi)}^{h_{\max }}$, is also the limiting process, as $y \rightarrow \infty$, when conditioning $\left(X_{t}, \varphi_{t}\right)_{t \geq 0}$, under $\mathrm{P}_{(e, \varphi)}$, on $\left\{H_{y}<\infty\right\}$. Furthermore, conditioning $\left(X_{t}, \varphi_{t}\right)_{t \geq 0}$, under $\mathrm{P}_{(e, \varphi)}^{h_{\max }}$, on $\left\{H_{0}=\infty\right\}$ yields the same result as the limit, as $y \rightarrow \infty$, of conditioning $\left(X_{t}, \varphi_{t}\right)_{t \geq 0}$ on $\left\{H_{y}<H_{0}\right\}$. In other words, the diagram in Figure 1 commutes. 


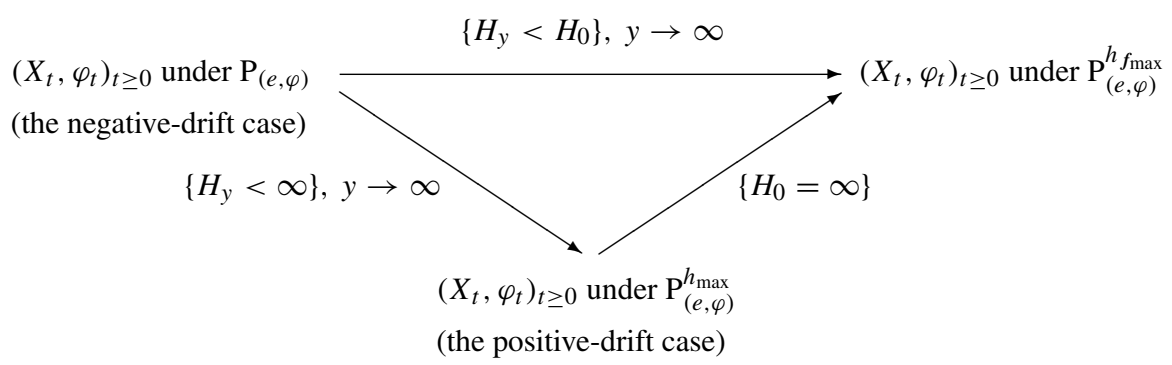

FIGURE 1: The conditioning of the process $\left(X_{t}, \varphi_{t}\right)_{t \geq 0}$ on the events $\left\{H_{y}<H_{0}\right\}, y \geq 0$, in the negativedrift case.

\section{The negative-drift case: conditioning $\left(\varphi_{t}\right)_{t \geq 0}$ to oscillate}

In this section, we condition the process $\left(\varphi_{t}\right)_{t \geq 0}$ with a negative drift to oscillate, and then condition the resulting oscillating process to stay nonnegative.

Let $\mathrm{P}_{(e, \varphi)}^{h}$ denote the $h$-transform of the measure $\mathrm{P}_{(e, \varphi)}$ by a positive, superharmonic function $h$ for the process $\left(X_{t}, \varphi_{t}\right)_{t \geq 0}$. We want to find an $h$ such that $\mathrm{P}_{(e, \varphi)}^{h}$ is honest, the process $\left(X_{t}\right)_{t \geq 0}$ is Markov under $\mathrm{P}_{(e, \varphi)}^{h}$, and the process $\left(\varphi_{t}\right)_{t \geq 0}$ oscillates under $\mathrm{P}_{(e, \varphi)}^{h}$. These desired properties of the function $h$ necessarily imply that it has to be harmonic.

First, we find the form of a positive, harmonic function $h$ for the process $\left(X_{t}, \varphi_{t}\right)_{t \geq 0}$ such that $\left(X_{t}\right)_{t \geq 0}$ is Markov under $\mathrm{P}_{(e, \varphi)}^{h}$.

Lemma 7.1. Suppose that a function $h$ is positive and harmonic for the process $\left(X_{t}, \varphi_{t}\right)_{t \geq 0}$ and that the process $\left(X_{t}\right)_{t \geq 0}$ is Markov under $\mathrm{P}_{(e, \varphi)}^{h}$. Then $h$ is of the form

$$
h(e, \varphi)=\mathrm{e}^{-\lambda \varphi} g(e), \quad(e, \varphi) \in E \times \mathbb{R},
$$

for some $\lambda \in \mathbb{R}$ and some positive vector $\mathrm{g}$ on $E$.

Proof. By the definition of $\mathrm{P}_{(e, \varphi)}^{h}$, for any $(e, \varphi) \in E \times \mathbb{R}$ and $t \geq 0$,

$$
\mathrm{P}_{(e, \varphi)}^{h}\left(X_{s}=e, 0 \leq s \leq t\right)=\frac{h(e, \varphi+v(e) t)}{h(e, \varphi)} \mathrm{P}_{(e, \varphi)}\left(X_{s}=e, 0 \leq s \leq t\right) .
$$

Since the process $\left(X_{t}\right)_{t \geq 0}$ is Markov under $\mathrm{P}_{(e, \varphi)}^{h}$, the probability $\mathrm{P}_{(e, \varphi)}^{h}\left(X_{s}=e, 0 \leq s \leq t\right)$ does not depend on $\varphi$. Thus, the right-hand side of the last equation does not depend on $\varphi$. Since $\mathrm{P}_{(e, \varphi)}\left(X_{s}=e, 0 \leq s \leq t\right)$ also does not depend on $\varphi$, because $\left(X_{t}\right)_{t \geq 0}$ is Markov under $\mathrm{P}_{(e, \varphi)}$, it follows that the ratio $h(e, \varphi+v(e) t) / h(e, \varphi)$ does not depend on $\varphi$. This implies that $h$ satisfies

$$
h(e, \varphi+y)=\frac{h(e, \varphi) h(e, y)}{h(e, 0)}, \quad e \in E, \varphi, y \in \mathbb{R} .
$$

Let $e \in E$ be fixed. Since the function $h$ is positive, we define a function $k_{e}(\varphi)$ by

$$
k_{e}(\varphi)=\log \left(\frac{h(e, \varphi)}{h(e, 0)}\right), \quad \varphi \in(0, \infty) .
$$


Then, by (7.1), the function $k_{e}$ is additive. In addition, it is measurable because $h$, as a harmonic function, is measurable. Therefore, it is also linear (see Aczel (1966)). It follows that the function $h$ is exponential, that is,

$$
h(e, \varphi)=h(e, 0) \mathrm{e}^{\lambda(e) \varphi}, \quad(e, \varphi) \in E_{0}^{+},
$$

for some function $\lambda(e)$ on $E$.

Hence, $h$ is continuously differentiable in $\varphi$, which, by Equation (3.4) of Jacka et al. (2005), implies that the $Q$-matrix of the process $\left(X_{t}\right)_{t \geq 0}$ under $\mathrm{P}_{(e, \varphi)}^{h}$ is given by

$$
\begin{aligned}
Q^{h}\left(e, e^{\prime}\right) & =\frac{h\left(e^{\prime}, \varphi\right)}{h(e, \varphi)} Q\left(e, e^{\prime}\right)+\frac{(\partial h / \partial \varphi)(e, \varphi)}{h(e, \varphi)} V\left(e, e^{\prime}\right) \\
& =\frac{h\left(e^{\prime}, 0\right)}{h(e, 0)} \mathrm{e}^{\left(\lambda(e)-\lambda\left(e^{\prime}\right)\right) \varphi} Q\left(e, e^{\prime}\right)+\lambda(e) V(e, e), \quad e, e^{\prime} \in E .
\end{aligned}
$$

However, because $\left(X_{t}\right)_{t \geq 0}$ is Markov under $\mathrm{P}_{(e, \varphi)}^{h}, \boldsymbol{Q}^{h}$ does not depend on $\varphi$. This implies that $\lambda(e)=-\lambda=$ const.

Finally, setting $g(e)=h(e, 0), e \in E$, proves the theorem.

The following theorem characterizes all positive, harmonic functions for the process $\left(X_{t}, \varphi_{t}\right)_{t \geq 0}$ with the properties stated at the beginning of the section.

Theorem 7.1. There exist exactly two positive, harmonic functions $h$ for the process $\left(X_{t}, \varphi_{t}\right)_{t \geq 0}$ such that the measure $\mathrm{P}_{(e, \varphi)}^{h}$ is honest and the process $\left(X_{t}\right)_{t \geq 0}$ is Markov under $\mathrm{P}_{(e, \varphi)}^{h}$. They are given by

$$
h_{\max }(e, \varphi)=\mathrm{e}^{-\alpha_{\max } \varphi} f_{\max }(e) \text { and } h_{\min }(e, \varphi)=\mathrm{e}^{-\beta_{\min } \varphi} g_{\min }(e) .
$$

Moreover,

(i) if the process $\left(\varphi_{t}\right)_{t \geq 0}$ drifts to $\infty$ then $h_{\max }=1$ and the process $\left(\varphi_{t}\right)_{t \geq 0}$, under $\mathrm{P}_{(e, \varphi)}^{h_{\min }}$, drifts to $-\infty$;

(ii) if the process $\left(\varphi_{t}\right)_{t \geq 0}$ drifts to $-\infty$ then $h_{\min }=1$ and the process $\left(\varphi_{t}\right)_{t \geq 0}$, under $\mathrm{P}_{(e, \varphi)}^{h_{\max }}$, drifts to $\infty$;

(iii) if the process $\left(\varphi_{t}\right)_{t \geq 0}$ oscillates then $h_{\max }=h_{\min }=1$.

Proof. We give a sketch of the proof. For the details, see Najdanovic (2003).

Let a function $h$ be positive and harmonic for the process $\left(X_{t}, \varphi_{t}\right)_{t \geq 0}$ and let the process $\left(X_{t}\right)_{t \geq 0}$ be Markov under $\mathrm{P}_{(e, \varphi)}^{h}$. Then, by Lemma 7.1, the function $h$ is of the form

$$
h(e, \varphi)=\mathrm{e}^{-\lambda \varphi} g(e), \quad(e, \varphi) \in E \times \mathbb{R},
$$

for some $\lambda \in \mathbb{R}$ and some vector $g$ on $E$.

Since the function $h$ is harmonic for the process $\left(X_{t}, \varphi_{t}\right)_{t \geq 0}$, it satisfies the equation $g h=\mathbf{0}$, where $g$, the generator of the process $\left(X_{t}, \varphi_{t}\right)_{t \geq 0}$, is given by Equation (3.4) of Jacka et al. (2005). Hence, $g h=(\boldsymbol{Q}+\boldsymbol{V}(\mathrm{d} / \mathrm{d} \varphi)) h=\mathbf{0}$ and $h(e, \varphi)=\mathrm{e}^{-\lambda \varphi} g(e)$ imply that $\boldsymbol{V}^{-1} \boldsymbol{Q} \boldsymbol{g}=\lambda \boldsymbol{g}$, that is, $\lambda$ is an eigenvalue of the matrix $\boldsymbol{V}^{-1} \boldsymbol{Q}$ and $\boldsymbol{g}$ is its associated eigenvector. In addition, by Lemma 3.6(i) of Jacka et al. (2005), the only positive eigenvectors of the matrix $\boldsymbol{V}^{-1} \boldsymbol{Q}$ are $\boldsymbol{f}_{\max }$ and $\boldsymbol{g}_{\min }$. Hence, $h(e, \varphi)=\mathrm{e}^{-\alpha_{\max } \varphi} f_{\max }(e)$ or $h(e, \varphi)=\mathrm{e}^{-\beta_{\min } \varphi} g_{\min }(e)$. 
The equality $G h=\mathbf{0}$ implies that the process $\left(h\left(X_{t}, \varphi_{t}\right)\right)_{t \geq 0}$ is a local martingale. Since the function $h(e, \varphi)=\mathrm{e}^{-\lambda \varphi} g(e)$ is bounded on every finite interval, the process $\left(h\left(X_{t}, \varphi_{t}\right)\right)_{t \geq 0}$ is a martingale. It follows that the measure $\mathrm{P}_{(e, \varphi)}^{h}$ is honest.

Let $Q^{h}$ be the $Q$-matrix of the process $\left(X_{t}\right)_{t \geq 0}$ under $\mathrm{P}_{(e, \varphi)}^{h}$. It can be shown that the eigenvalues of the matrix $\boldsymbol{V}^{-1} \boldsymbol{Q}^{h_{\min }}$ coincide with the eigenvalues of the matrix $\boldsymbol{V}^{-1}\left(\boldsymbol{Q}-\beta_{\min } \boldsymbol{I}\right)$, and that the eigenvalues of the matrix $\boldsymbol{V}^{-1} \boldsymbol{Q}^{h_{\max }}$ coincide with the eigenvalues of the matrix $\boldsymbol{V}^{-1}\left(\boldsymbol{Q}-\alpha_{\max } \boldsymbol{I}\right)$. These facts together with Equation (3.6) of Jacka et al. (2005) prove statements (i)-(iii).

By Theorem 7.1(ii) there does not exist a positive function $h$, harmonic for the process $\left(X_{t}, \varphi_{t}\right)_{t \geq 0}$, such that $\mathrm{P}_{(e, \varphi)}^{h}$ is honest, the process $\left(X_{t}\right)_{t \geq 0}$ is Markov under $\mathrm{P}_{(e, \varphi)}^{h}$, and the process $\left(\varphi_{t}\right)_{t \geq 0}$ oscillates under $\mathrm{P}_{(e, \varphi)}^{h}$ (we recall that initially the process $\left(\varphi_{t}\right)_{t \geq 0}$ drifts to $-\infty$ under $\left.\mathrm{P}_{(e, \varphi)}\right)$. However, we can look for a positive, space-time-harmonic function $h$ for $\left(X_{t}, \varphi_{t}\right)_{t \geq 0}$ that has the desired properties. We restrict ourselves to the set of such continuous functions.

Lemma 7.2. Suppose that a function $h$ is positive, continuous, and space-time harmonic for the process $\left(X_{t}, \varphi_{t}\right)_{t \geq 0}$ and that the process $\left(X_{t}\right)_{t \geq 0}$ is (time-homogeneous) Markov under $\mathrm{P}_{(e, \varphi)}^{h}$. Then $h$ is of the form

$$
h(e, \varphi, t)=\mathrm{e}^{-\alpha t} \mathrm{e}^{-\beta \varphi} g(e), \quad(e, \varphi) \in E \times \mathbb{R},
$$

for some $\alpha, \beta \in \mathbb{R}$ and some vector $\mathrm{g}$ on $E$.

Proof. Define $u=\min _{e \in E} v(e)<0, v=\max _{e \in E} v(e)>0$, and the cone

$$
C=\{(0,0)\} \cup\{(s, y): y>0, u s<y<v s\} .
$$

By the definition of the $h$-transform, for any $(e, \varphi) \in E \times \mathbb{R}, t \geq 0$, and any $(s, y) \in C$,

$$
\mathrm{P}_{(e, \varphi, t)}^{h}\left(X_{t+s}=e, \varphi_{t+s} \in \varphi+\mathrm{d} y\right)=\frac{h(e, \varphi+\mathrm{d} y, t+s)}{h(e, \varphi, t)} \mathrm{P}_{(e, \varphi, t)}\left(X_{t+s}=e, \varphi_{t+s} \in \varphi+\mathrm{d} y\right) .
$$

Since the process $\left(X_{t}\right)_{t \geq 0}$ is time-homogeneous Markov under $\mathrm{P}_{(e, \varphi)}^{h}$, we have

$$
\mathrm{P}_{(e, \varphi, t)}^{h}\left(X_{t+s}=e, \varphi_{t+s} \in \varphi+\mathrm{d} y\right)=\mathrm{P}_{(e, 0,0)}^{h}\left(X_{s}=e, \varphi_{s} \in \mathrm{d} y\right) .
$$

Similarly,

$$
\mathrm{P}_{(e, \varphi, t)}\left(X_{t+s}=e, \varphi_{t+s} \in \varphi+\mathrm{d} y\right)=\mathrm{P}_{(e, 0,0)}\left(X_{s}=e, \varphi_{s} \in \mathrm{d} y\right) .
$$

Therefore, it follows from (7.2) that the ratio $h(e, \varphi+y, t+s) / h(e, \varphi, t)$ does not depend on $\varphi$ and $t$ (for $(s, y) \in C$ ). This implies that $h$ satisfies

$$
h(e, \varphi+y, t+s)=\frac{h(e, \varphi, t) h(e, y, s)}{h(e, 0,0)}, \quad e \in E, \varphi \in \mathbb{R}, t \geq 0,(s, y) \in C .
$$

Since the function $h$ is positive, we can define a function $k(e, \varphi, t)$ by

$$
k(e, \varphi, t)=\log \left(\frac{h(e, \varphi, t)}{h(e, 0,0)}\right), \quad(e, \varphi, t) \in E_{0}^{+} \times[0, \infty) .
$$


Then, by (7.3),

$$
k(e, \varphi+y, t+s)=k(e, \varphi, t)+k(e, y, s), \quad e \in E,(t, \varphi),(s, y) \in C .
$$

Now fix a ray of the form $(t, c t)$ in $C$. It follows, by the same argument as in the proof of Lemma 7.1 , that there exists a $\lambda(\cdot, \cdot)$ such that

$$
h(e, \varphi+c s, t+s)=\mathrm{e}^{\lambda(e, c) s} h(e, \varphi, t),
$$

and it is then straightforward (by considering different routes from $(t, \varphi)$ to $(t+s, \varphi+y))$ to see that there exist a $\lambda(\cdot)$ and a $\mu(\cdot)$ such that

$$
h(e, \varphi, t)=\mathrm{e}^{\lambda(e) t+\mu(e) \varphi} h(e, 0,0) .
$$

That $\lambda$ and $\mu$ are independent of $e$ then follows in the same way as in the proof of Lemma 7.1.

Theorem 7.2. All positive, continuous, space-time-harmonic functions $h$ for the process $\left(X_{t}, \varphi_{t}\right)_{t \geq 0}$, such that $\mathrm{P}_{(e, \varphi)}^{h}$ is honest and $\left(X_{t}\right)_{t \geq 0}$ is Markov under $\mathrm{P}_{(e, \varphi)}^{h}$, are of the form

$$
h(e, \varphi, t)=\mathrm{e}^{-\alpha t} \mathrm{e}^{-\beta \varphi} g(e), \quad(e, \varphi, t) \in E \times \mathbb{R} \times[0, \infty),
$$

where, for a fixed $\beta \in \mathbb{R}, \alpha \equiv \alpha(\beta)$ is the Perron-Frobenius eigenvalue and $\boldsymbol{g}$ is the right Perron-Frobenius eigenvector of the matrix $\boldsymbol{Q}-\beta \boldsymbol{V}$.

Moreover, there exists a unique $\beta_{0} \in \mathbb{R}$ such that, under $\mathrm{P}_{(e, \varphi)}^{h}$,

- $\left(\varphi_{t}\right)_{t \geq 0}$ drifts to $\infty$ if and only if $\beta<\beta_{0}$,

- $\left(\varphi_{t}\right)_{t \geq 0}$ oscillates if and only if $\beta=\beta_{0}$,

- $\left(\varphi_{t}\right)_{t \geq 0}$ drifts to $-\infty$ if and only if $\beta>\beta_{0}$,

and $\beta_{0}$ is determined by the equation $\alpha^{\prime}\left(\beta_{0}\right)=0$.

Proof. We again give only a sketch of the proof. For the details, see Najdanovic (2003).

Let a function $h$ be positive, continuous, and space-time harmonic for the process $\left(X_{t}, \varphi_{t}\right)_{t \geq 0}$, and let the process $\left(X_{t}\right)_{t \geq 0}$ be Markov under $\mathrm{P}_{(e, \varphi)}^{h}$. Then, by Lemma 7.2, the function $h$ is of the form

$$
h(e, \varphi, t)=\mathrm{e}^{-\alpha t} \mathrm{e}^{-\beta \varphi} g(e), \quad(e, \varphi, t) \in E \times \mathbb{R} \times[0, \infty),
$$

for some $\alpha, \beta \in \mathbb{R}$ and some vector $\boldsymbol{g}$ on $E$.

Since the function $h$ is harmonic for the process $\left(X_{t}, \varphi_{t}\right)_{t \geq 0}$, it satisfies the equation $\mathcal{A} h=\mathbf{0}$, where $\mathcal{A}$, the generator of the process $\left(X_{t}, \varphi_{t}\right)_{t \geq 0}$, is given by Equation (3.5) of Jacka et al. (2005). Hence, $\mathcal{A} h=(\boldsymbol{Q}+\boldsymbol{V}(\mathrm{d} / \mathrm{d} \varphi)+\boldsymbol{I}(\mathrm{d} / \mathrm{d} t)) h=\mathbf{0}$ and $h(e, \varphi, t)=\mathrm{e}^{-\alpha t} \mathrm{e}^{-\beta \varphi} g(e)$ imply that $(\boldsymbol{Q}-\beta \boldsymbol{V}) \boldsymbol{g}=\alpha \boldsymbol{g}$, i.e. that $\alpha$ is an eigenvalue of the matrix $\boldsymbol{Q}-\beta \boldsymbol{V}$ and $\boldsymbol{g}$ is its associated eigenvector. In addition, by Lemma 3.1 of Jacka et al. (2005), the matrix $\boldsymbol{Q}-\beta \boldsymbol{V}$ is irreducible and essentially nonnegative. By the Perron-Frobenius theorem, the only positive eigenvector of an irreducible and essentially nonnegative matrix is its Perron-Frobenius eigenvector. Thus, $\alpha$ and $\boldsymbol{g}$ are, respectively, the Perron-Frobenius eigenvalue and eigenvector of the matrix $\boldsymbol{Q}-\beta \boldsymbol{V}$.

The equation $\mathcal{A} h=\mathbf{0}$ implies that the process $h\left(X_{t}, \varphi_{t}, t\right)_{t \geq 0}$ is a local martingale. Since the function $h(e, \varphi, t)=\mathrm{e}^{-\alpha t} \mathrm{e}^{-\beta \varphi} g(e)$ is bounded on every finite interval, the process $h\left(X_{t}, \varphi_{t}, t\right)_{t \geq 0}$ is a martingale. It follows that the measure $\mathrm{P}_{(e, \varphi)}^{h}$ is honest.

For a fixed $\beta \in \mathbb{R}$, let $h(e, \varphi, t)=\mathrm{e}^{-\alpha(\beta) t} \mathrm{e}^{-\beta \varphi}[\boldsymbol{g}(\beta)](e)$, where $\alpha(\beta)$ and $\boldsymbol{g}(\beta)$ are respectively the Perron-Frobenius eigenvalue and right eigenvector of the matrix $\boldsymbol{Q}-\beta \boldsymbol{V}$. Let $\boldsymbol{\mu}_{\beta}$ 
denote the invariant measure of the process $\left(X_{t}\right)_{t \geq 0}$ under $\mathrm{P}_{(e, \varphi)}^{h}$, and let $\boldsymbol{g}^{\text {left }}(\beta)$ denote the left eigenvector of the matrix $\boldsymbol{Q}-\beta \boldsymbol{V}$. It can then be shown that $\boldsymbol{\mu}_{\beta} \boldsymbol{V} \mathbf{1}=\boldsymbol{g}^{\text {left }}(\beta) \boldsymbol{V} \boldsymbol{g}(\beta)$. Since $\left[\boldsymbol{g}^{\text {left }}(\beta)\right](e)[\boldsymbol{g}(\beta)](e)>0$ for every $e \in E$, Lemma 3.9 and Equation (3.6) of Jacka $e t$ al. (2005) imply the statement of the second part of the theorem.

By Theorem 7.2, there exists exactly one positive, continuous, space-time-harmonic function $h$ for the process $\left(X_{t}, \varphi_{t}\right)_{t \geq 0}$ with the desired properties, and it is given by

$$
h_{0}(e, \varphi, t)=\mathrm{e}^{-\alpha_{0} t} \mathrm{e}^{-\beta_{0} \varphi} g_{0}(e), \quad(e, \varphi, t) \in E \times \mathbb{R} \times[0, \infty),
$$

where $\alpha_{0}=\alpha\left(\beta_{0}\right)$ and $\boldsymbol{g}_{0}$ is the Perron-Frobenius eigenvector of $\boldsymbol{Q}-\beta_{0} \boldsymbol{V}$. For a fixed $(e, \varphi) \in E_{0}^{+}$, let a measure $\mathrm{P}_{(e, \varphi)}^{h_{0}}$ be defined by

$$
\mathrm{P}_{(e, \varphi)}^{h_{0}}(A)=\frac{\mathrm{E}_{(e, \varphi)}\left(1(A) h_{0}\left(X_{t}, \varphi_{t}, t\right)\right)}{h_{0}(e, \varphi, 0)}, \quad A \in \mathcal{F}_{t}, t \geq 0,
$$

and let $\mathrm{E}_{(e, \varphi)}^{h_{0}}$ denote the associated expectation operator. Then the process $\left(X_{t}\right)_{t \geq 0}$, under $\mathrm{P}_{(e, \varphi)}^{h_{0}}$, is Markov with $Q$-matrix $\boldsymbol{Q}^{0}$ given by

$$
Q^{0}\left(e, e^{\prime}\right)=\frac{g_{0}\left(e^{\prime}\right)}{g_{0}(e)}\left[\boldsymbol{Q}-\alpha_{0} \boldsymbol{I}-\beta_{0} \boldsymbol{V}\right]\left(e, e^{\prime}\right), \quad e, e^{\prime} \in E,
$$

and, by Theorem 7.2, under $\mathrm{P}_{(e, \varphi)}^{h_{0}}$ the process $\left(\varphi_{t}\right)_{t \geq 0}$ oscillates.

The aim now is to condition $\left(X_{t}, \varphi_{t}\right)_{t \geq 0}$, under $\mathrm{P}_{(e, \varphi)}^{h_{0}}$, on the event that $\left(\varphi_{t}\right)_{t \geq 0}$ stays nonnegative. The following theorem determines the law of this new conditioned process.

Theorem 7.3. For a fixed $(e, \varphi) \in E_{0}^{+}$, let a measure $\mathrm{P}_{(e, \varphi)}^{h_{0}, h_{r}^{0}}$ be defined by

$$
\mathrm{P}_{(e, \varphi)}^{h_{0}, h_{r}^{0}}(A)=\frac{\mathrm{E}_{(e, \varphi)}^{h_{0}}\left(1(A) h_{\boldsymbol{r}}^{0}\left(X_{t}, \varphi_{t}\right) 1\left(t<H_{0}\right)\right)}{h_{\boldsymbol{r}}^{0}(e, \varphi)}, \quad A \in \mathcal{F}_{t}, t \geq 0,
$$

where the function $h_{\boldsymbol{r}}^{0}$ is given by

$$
h_{\boldsymbol{r}}^{0}(e, y)=\left[\mathrm{e}^{-y \boldsymbol{V}^{-1} \boldsymbol{Q}^{0}} \boldsymbol{J}_{1} \boldsymbol{\Gamma}_{2} \boldsymbol{r}^{0}\right](e), \quad(e, y) \in E \times \mathbb{R},
$$

and $\boldsymbol{r}^{0}$ satisfies $\boldsymbol{V}^{-1} \boldsymbol{Q}^{0} \boldsymbol{r}^{0}=\mathbf{1}$. Thus, $\mathrm{P}_{(e, \varphi)}^{h_{0}, h_{r}^{0}}$ is a probability measure.

In addition, for $t \geq 0$ and $A \in \mathcal{F}_{t}$,

$$
\mathrm{P}_{(e, \varphi)}^{h_{0}, h_{r}^{0}}(A)=\lim _{y \rightarrow \infty} \mathrm{P}_{(e, \varphi)}^{h_{0}}\left(A \mid H_{y}<H_{0}\right)=\lim _{T \rightarrow \infty} \mathrm{P}_{(e, \varphi)}^{h_{0}}\left(A \mid H_{0}>T\right)
$$

and

$$
\mathrm{P}_{(e, \varphi)}^{h_{0}, h_{r}^{0}}(A)=\mathrm{P}_{(e, \varphi)}^{h_{r}^{0}}(A),
$$

where $\mathrm{P}_{(e, \varphi)}^{h^{0}}$ is as defined in Theorem 2.2 of Jacka et al. (2005).

Proof. By (7.4), for $t \geq 0$ and $A \in \mathcal{F}_{t}$,

$$
\begin{aligned}
\mathrm{P}_{(e, \varphi)}^{h_{0}, h_{r}^{0}}(A) & =\frac{\mathrm{E}_{(e, \varphi)}\left(1(A) h_{0}\left(X_{t}, \varphi_{t}, t\right) h_{\boldsymbol{r}}^{0}\left(X_{t}, \varphi_{t}\right) 1\left(t<H_{0}\right)\right)}{h_{0}(e, \varphi, 0) h_{r}^{0}(e, \varphi)} \\
& =\frac{\mathrm{E}_{(e, \varphi)}\left(1(A) h_{\boldsymbol{r}^{0}}\left(X_{t}, \varphi_{t}, t\right) 1\left(t<H_{0}\right)\right)}{h_{\boldsymbol{r}^{0}}(e, \varphi, t)}
\end{aligned}
$$




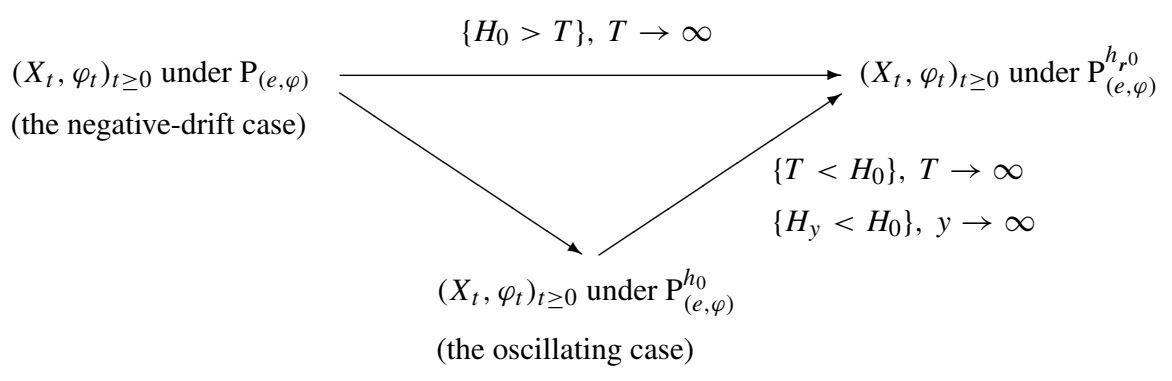

FIGURE 2: The conditioning of the process $\left(X_{t}, \varphi_{t}\right)_{t \geq 0}$ on the events $\left\{H_{0}>T\right\}, T \geq 0$, in the negativedrift case.

where

$$
h_{\boldsymbol{r}^{0}}(e, \varphi, t)=h_{0}(e, \varphi, t) h_{\boldsymbol{r}}^{0}(e, \varphi)=\mathrm{e}^{-\alpha_{0} t} \mathrm{e}^{-\beta_{0} \varphi}\left[\boldsymbol{G}_{0} \mathrm{e}^{-\varphi \boldsymbol{V}^{-1} \boldsymbol{Q}^{0}} \boldsymbol{J}_{1} \boldsymbol{\Gamma}_{2}^{0} \boldsymbol{r}^{0}\right](e)
$$

is as defined in Theorem 2.2 of Jacka et al. (2005). By Lemma 5.1(i) of Jacka et al. (2005), the function $h_{\boldsymbol{r}^{0}}(e, \varphi, t)$ is positive and, by Lemma 5.5 of Jacka et al. (2005), the function $h_{\boldsymbol{r}^{0}}(e, \varphi, t)$ is space-time harmonic for the process $\left(X_{t}, \varphi_{t}, t\right)_{t \geq 0}$. Thus, $\mathrm{P}_{(e, \varphi)}^{h_{0}, h_{r}^{0}}$ is a probability measure and, by the definition of the measure $\mathrm{P}_{(e, \varphi)}^{r^{0}}$ in Theorem 2.2 of Jacka et al. (2005),

$$
\mathrm{P}_{(e, \varphi)}^{h_{0}, h_{r}^{0}}(A)=\mathrm{P}_{(e, \varphi)}^{h^{0}}(A), \quad A \in \mathcal{F}_{t}, t \geq 0 .
$$

In addition, by Equation (3.6) and Lemma 3.11 of Jacka et al. (2005), the $Q$-matrix $Q^{0}$ of the process $\left(X_{t}\right)_{t \geq 0}$, under $\mathrm{P}_{(e, \varphi)}^{h_{0}}$, is conservative and irreducible and the process $\left(\varphi_{t}\right)_{t \geq 0}$, under $\mathrm{P}_{(e, \varphi)}^{h_{0}}$, oscillates. Thus, by our Theorem 2.1 and Theorem 2.1 of Jacka et al. (2005), $\mathrm{P}_{(e, \varphi)}^{h_{0}, h_{r}^{0}}$ denotes the law of $\left(X_{t}, \varphi_{t}\right)_{t \geq 0}$, under $\mathrm{P}_{(e, \varphi)}^{h_{0}}$, conditioned on $\left\{H_{0}=\infty\right\}$, and, for any $t \geq 0$ and $A \in \widetilde{F}_{t}$,

$$
\mathrm{P}_{(e, \varphi)}^{h_{0}, h_{r}^{0}}(A)=\lim _{y \rightarrow \infty} \mathrm{P}_{(e, \varphi)}^{h_{0}}\left(A \mid H_{y}<H_{0}\right)=\lim _{T \rightarrow \infty} \mathrm{P}_{(e, \varphi)}^{h_{0}}\left(A \mid H_{0}>T\right) .
$$

To conclude, let us summarize the results of this section. In the negative-drift case, making the $h$-transform of the process $\left(X_{t}, \varphi_{t}, t\right)_{t \geq 0}$ with the function $h_{0}(e, \varphi, t)=\mathrm{e}^{-\alpha_{0} t} \mathrm{e}^{-\beta_{0} \varphi} g_{0}(e)$ yields the probability measure $\mathrm{P}_{(e, \varphi)}^{h_{0}}$ such that $\left(X_{t}\right)_{t \geq 0}$, under $\mathrm{P}_{(e, \varphi)}^{h_{0}}$, is Markov and $\left(\varphi_{t}\right)_{t \geq 0}$, under $\mathrm{P}_{(e, \varphi)}^{h_{0}}$, oscillates. Then the law of $\left(X_{t}, \varphi_{t}\right)_{t \geq 0}$, under $\mathrm{P}_{(e, \varphi)}^{h_{0}}$, conditioned on the event $\left\{H_{0}=\infty\right\}$, is equal to $\mathrm{P}_{(e, \varphi)}^{h_{0}, h_{r}^{0}}=\mathrm{P}_{(e, \varphi)}^{h_{r}{ }^{0}}$. On the other hand, by Theorem 2.2 of Jacka et al. (2005), under the condition that all nonzero eigenvalues of the matrix $V^{-1} Q^{0}$ are simple, $\mathrm{P}_{(e, \varphi)}^{h^{0}}$ is the limiting law as $T \rightarrow \infty$ of the process $\left(X_{t}, \varphi_{t}\right)_{t \geq 0}$, under $\mathrm{P}_{(e, \varphi)}^{r^{0}}$, conditioned on $\left\{H_{0}>T\right\}$. Hence, under the condition that all nonzero eigenvalues of the matrix $\boldsymbol{V}^{-1} \boldsymbol{Q}^{0}$ are simple, the diagram in Figure 2 commutes.

\section{References}

Aczel, J. (1966). Lectures on Functional Equations and Their Applications. Academic Press, New York.

Bertoin, J. And Doney, R. A. (1994). On conditioning a random walk to stay nonnegative. Ann. Prob. 22, $2152-2167$.

JACKA, S. D., LAZIC, Z. AND WARren, J. (2005). Conditioning an additive functional of a Markov chain to stay nonnegative. I. Survival for a long time. Adv. Appl. Prob. 37, 1015-1034.

Najdanovic, Z. (2003). Conditioning a Markov chain upon the behaviour of an additive functional. Doctoral Thesis, University of Warwick.

SenetA, E. (1981). Nonnegative Matrices and Markov Chains, 2nd edn. Springer, New York. 